8-27-2012

\title{
Rigid Finite Element Model of a Cracked Rotor
}

\author{
Zbigniew Kulesza \\ Bialystok University of Technology, z.kulesza@pb.edu.pl \\ Jerzy T. Sawicki \\ Cleveland State University, j.sawicki@csuohio.edu
}

Follow this and additional works at: https://engagedscholarship.csuohio.edu/enme_facpub

Part of the Mechanical Engineering Commons

How does access to this work benefit you? Let us know!

\section{Publisher's Statement}

NOTICE: this is the author's version of a work that was accepted for publication in Journal of Sound and Vibration. Changes resulting from the publishing process, such as peer review, editing, corrections, structural formatting, and other quality control mechanisms may not be reflected in this document. Changes may have been made to this work since it was submitted for publication. A definitive version was subsequently published in Journal of Sound and Vibration, 331, 18, (08-27-2012); 10.1016/j.jsv.2012.04.014

\section{Original Citation}

Zbigniew Kulesza, Jerzy T. Sawicki. (2012). Rigid Finite Element Model of a Cracked Rotor. Journal of Sound and Vibration, 331(18), 4145-4169, doi: dx.doi.org/10.1016/j.jsv.2012.04.014.

This Article is brought to you for free and open access by the Mechanical Engineering Department at EngagedScholarship@CSU. It has been accepted for inclusion in Mechanical Engineering Faculty Publications by an authorized administrator of EngagedScholarship@CSU. For more information, please contact library.es@csuohio.edu. 


\section{Rigid finite element model of a cracked rotor}

\section{Zbigniew Kulesza ${ }^{\text {a,* }}$, Jerzy T. Sawicki ${ }^{\text {b }}$}

${ }^{a}$ Faculty of Mechanical Engineering, Bialystok University of Technology, ul. Wiejska 45C, 15-351 Bialystok, Poland

${ }^{\mathrm{b}}$ Center for Rotating Machinery Dynamics and Control (RoMaDyC), Fenn College of Engineering, Cleveland State University, Cleveland, OH 44115-2214. USA

\section{Introduction}

The problem of modeling of cracked shafts has been the subject of interest of many research centers for the past four decades. This is mainly due to the fact that shaft cracks, which may appear in rotating machines during their operation, are serious problems and may lead to catastrophic accidents if not detected early. Transverse cracks in rotors occur due to cyclic loading, creep, stress corrosion, and other mechanisms to which rotating shafts are subjected. An early crack warning can considerably extend the durability of rotating machines, increasing their reliability at the same time.

Known approaches to shaft crack modeling can be divided into three main categories [1]: local stiffness reduction, discrete spring models, and complex models in two or three dimensions.

The simplest methods aim to reduce the local stiffness of the shaft near the location of the crack [2-6]. The reduction of stiffness is in some degree proportional to the depth of the crack. This is usually achieved by reducing the second moment of area of the shaft cross section $[4,6]$.

\footnotetext{
"Corresponding author. Tel.: +48 8574692 34; fax: +48 857469210.

E-mail address: z.kulesza@pb.edu.pl (Z. Kulesza).
} 


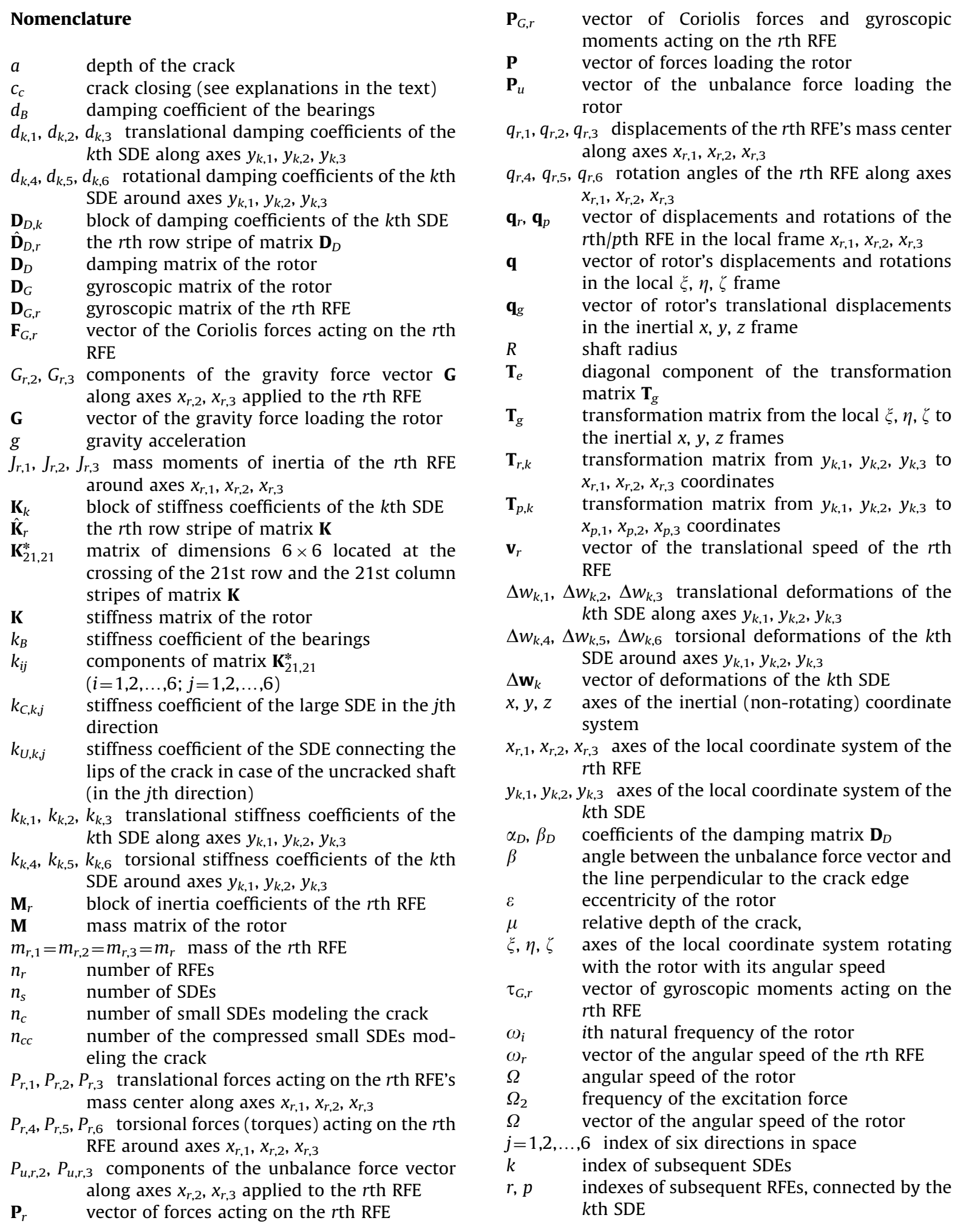

The reduced stiffness may remain constant in the fixed angular direction, leading to linear equations of motion for the cracked shaft, similar to the ones for an asymmetric shaft [7,8]. This corresponds to the so called fully open cracks (slotted or notch cracks). However, many experimental results show that, for the majority of rotating cracked shafts, the so called 
breathing mechanism should be included. This mechanism, which manifests itself in periodical stiffness changes, resulting from periodical opening and closing of the crack lips, may be introduced by relatively simple models of Grabowski [3], Gasch [2], Mayes and Davies [4], or by more complex models of Ostachowicz and Krawczuk [9], and Darpe et al. [10], which actually fall into the third category.

Grabowski [3] suggested switching of the stiffness values from those of an uncracked rotor (closed crack state) to those of a cracked rotor (fully open state) at a particular rotor angular position. Grabowski's model can be applied, when the weight dominance of the external load is assumed [11-13]. In this situation, the constant static deflection of the rotor may be neglected, and only the dynamic, vibration response is calculated, simplifying the analysis.

Gasch [14] modified this approach, demanding that the switching takes place not at a particular angular position, but only when there is a change in the sign of rotor deflection in the direction perpendicular to the crack edge. His modification, which is often referred to as the hinge model, extends possible applications to other rotors, not only to the weight-dominant ones.

Mayes and Davies [4] suggested a sinusoidal stiffness change to model the breathing in a more sensible way, as a rotor crack is expected to open and close gradually due to external loads. Sawicki et al. [15] combined the Gash, Mayes and Davies models, introducing an additional angle $\psi$ into the Jeffcott rotor model, in order to determine the amount of crack opening, when the rotor weight-dominance is ignored. Smoother changes from the open to close state of the crack can be obtained by using Fourier expansion for the so called crack steering function, which is especially applicable when approximated methods, such as harmonic balance [12] or multiple scales [16] are applied in order to calculate the response of the cracked rotor. Two new crack steering functions have been recently developed by Al-Shudeifat and Butcher [29] who used them for a more exact evaluation of the cracked shaft stiffness changes.

Along with the progressive development of the finite element method (FEM), FEM models of rotors, bearings and other components have been introduced for rotordynamic calculations [17]. First finite element models of the rotating shaft cracks were introduced by fundamental works of Dimarogonas and Paipetis [18], who starting from the fracture mechanics, obtained a full $6 \times 6$ flexibility matrix for a transverse open surface crack on a shaft. Papadopoulos and Dimarogonas [19] derived the flexibility matrix for the finite shaft element with an open crack. Ostachowicz and Krawczuk [9] used the finite element model with a modified stiffness matrix of a beam accounting for the effect of crack and considering all but axial degree of freedom.

Darpe et al. [10] provided more detailed and complete derivations of the flexibility matrix of a cracked rotor segment starting from Castigliano's theorem. They introduced the original model of the crack breathing mechanism, in which the extent of crack opening is determined by calculating the values of compressive stresses at the crack edge (i.e., not the stresses themselves, but the so called stress intensity factors (SIFs) along the crack edge are calculated at each simulation step in order to determine the state of the crack). The closed part of the crack surface is delimited by a boundary, the so called crack closure line $(\mathrm{CCL})$ represented by a segment orthogonal to the crack edge. Vare and Andrieux [20] extended this concept by introducing a more realistic true breathing mechanism, which can be calculated by means of 3D finite models in a nonlinear approach. The open and closed areas of the crack cross section were determined by calculating the compressive stresses at each point of the cracked area. As the calculations of the true breathing mechanism were very time consuming, Bachschmid et al. [21] suggested a simplified model, which assumed linear stress and strain distribution, as well as transient thermal stresses that can arise in rotating shafts. The results they obtained were very accurate, yet the calculation procedure was not so trivial.

The characteristic feature of the cracked shaft finite element, modeled using fracture mechanics approach $[9,10,18,19]$ is the presence of additional components that appear beyond the main diagonal of the stiffness matrix. This may lead to the couplings between torsional, bending and longitudinal modes of vibration. The appearance of the coupled vibrations may be used for shaft crack detection. Ostachowicz and Krawczuk [9], Papadopoulos and Dimarogonas [19] reported the possibility to use the torsional-bending, while Darpe et al. [22] reported using bending-longitudinal coupled vibrations for such purposes. Unfortunately, these methods have not been widely employed due to very low amplitudes of the induced, coupled vibrations.

The models that fall into the second category, i.e. discrete spring models of the crack are met relatively rarely. Vaziri and Nayeb-Hashemi [23] studied a circumferentially cracked shaft subjected to cyclic torsion. They presented a model consisting of two shaft segments connected by a torsional spring and a torsional damper. The spring and the damper represented the local flexibility and the local energy loss of the crack region, respectively. However, the stiffness and damping for other modes (bending and torsional) of the shaft deflections were not included. El Arem [24] suggested a model in which the transverse cracked section was replaced by two lumped nonlinear flexural and shearing springs. He considered a 3D finite element model of a shaft in which the opening and closure of the crack were governed by the normal stress on the crack lips. He found that the influence of shearing effects on the breathing mechanism of the crack was negligible when compared to this of the flexural moments.

The present article recommends the discrete mass-spring-damper model of the cracked shaft, utilizing the approach known as the rigid finite element (RFE) method [25]. The rigid finite element method is based on a completely different approach than the classical finite element method (FEM). The idea is to discretize the given mechanical structure (such as a bar, beam, frame or shell) into rigid elements containing inertial features of the structure. These rigid elements are connected by massless and non-dimensional spring-damping elements (SDEs). The method has been successfully applied for the dynamic analysis of mechanisms, machine tools, cranes, ship drive systems, and even ship hulls [25]. It is mainly 
due to many advantages of the RFE method, which include: simplicity (the model of a very complex mechanical structure can be obtained quickly and intuitively), a uniform approach to describe rigid and flexible bodies, the numerical effectiveness, and the possibility to analyze both small and large deformations. Lately, the method has been improved and extended by Wittbrodt et al. [26], who used it for successful dynamic calculations of flexible multibody systems with changing configurations, such as robot manipulators.

The rigid finite element method can be classified into a group of well known lumped parameter methods [27]. The modification of the lumped parameters method, namely the transfer matrices method (or Myklestadt-Prohl method), has been widely used for modeling rotating machines for years [27]. Like the transfer matrices method, the RFE method allows one to create the model of the given machine in a very systematic and intuitive way, yet the RFE method does not seem to have so many limitations as its earlier ancestor.

The idea of applying the rigid finite element method for modeling driving shafts is not new; e.g. natural frequencies of warship/trawler driving shafts were calculated this way [25]. However, to the authors' knowledge, it is the first time that RFEs will be used for modeling the crack in the rotating shaft. Similarly to the previous works of Bachschmid et al. [21], the open and closed areas are determined by calculating deformations at selected points of the crack surface, yet the surface does not have to be divided into the refined mesh, as the deformations are determined in several spring-damping elements (SDEs) connecting the two segments of the shaft. The authors believe that such an approach is simpler and less time-consuming than the previous ones $[20,21]$, and that several interesting problems, particularly concerning the breathing mechanism, can be explained and better understood using it.

The article presents the rigid finite element model of a given rotating machine, paying particular interest to the problem of modeling the crack in the shaft. The results of calculations of the uncracked free-free rotor are compared with the experimental data and with the results obtained with other software. Next, transient analysis is performed and frequency response of the rotor is calculated. Some remarks concerning the possible coupling mechanisms between torsional, bending and longitudinal vibrations are discussed. The influence of friction between the lips of the crack on the rotor vibration response is investigated.

\section{Fundamentals of the rigid finite element (RFE) method}

Using the rigid finite element method [25], a given structure is divided into a defined number of lumped elements: $n_{r}$ non-deformable, rigid bodies (rigid finite elements (RFEs)) connected with $n_{s}$ spring-damping elements (SDEs) (see Fig. 1). The way RFEs connect with SDEs is arbitrary. Rigid finite elements are numbered from 1 to $n_{r}$, and spring-damping elements-from 1 to $n_{s}$.

Rigid finite elements are characterized by their masses and mass moments of inertia, while spring-damping elements-by their stiffness and damping coefficients. The masses of spring-damping elements are neglected. Static

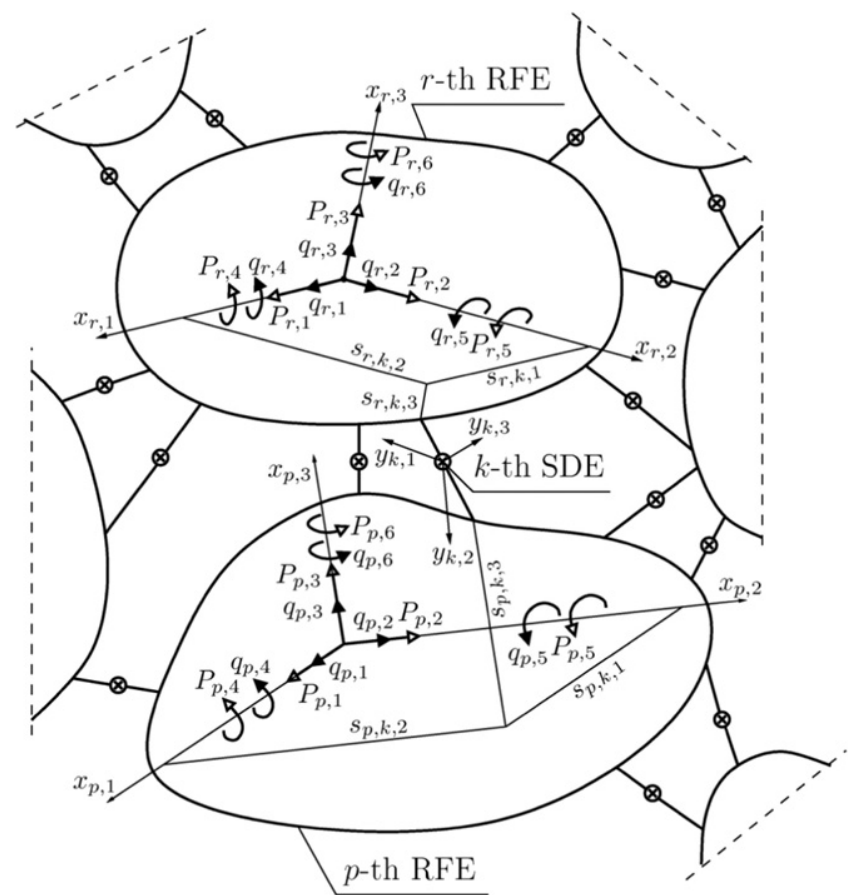

Fig. 1. Structure model consisting of RFEs connected by SDEs; closed arrows—general displacements, open arrows-general forces (based on [25]). 
characteristic of each spring-damping element is linear, which means that the force loading the element is the sum of two forces: one that is proportional to deformation (stiffness), and another that is proportional to deformation velocity (damping). Spring-damping elements can be loaded with translational forces, as well as with pairs of forces (torques).

The motion of each RFE is considered in an independent coordinate system with three main axes $x_{r, 1}, x_{r, 2}, x_{r, 3}$. The center of the system is located at the mass center of a given element. At a standstill, axes $x_{r, 1}, x_{r, 2}$ and $x_{r, 3}$ coincide with three principal axes of the element, i.e. with the axes around which deviatory moments are zero.

Similarly, the motion of each SDE is considered in an independent coordinate system defined by three main axes $y_{k, 1}$, $y_{k, 2}, y_{k, 3}$. The main axes of the SDE should be chosen in such a way that the force acting along each axis causes only a translational deformation in a direction along the axis. Similarly, the pair of forces acting around each coordinate axis should cause only a torsional deformation around the axis.

Each rigid finite element is defined by a block of inertia coefficients. This block has a form of a diagonal matrix, because $x_{r, 1}, x_{r, 2}$ and $x_{r, 3}$ are principal axes of the RFE. For spatial systems the block of inertia coefficients for the $r$ th RFE is, as follows [25]:

$$
\left.\mathbf{M}_{r}=\operatorname{diag}\left[\begin{array}{llllll}
m_{r, 1} & m_{r, 2} & m_{r, 3} & J_{r, 1} & J_{r, 2} & J_{r, 3}
\end{array}\right]\right) .
$$

Here, $\operatorname{diag}(\mathbf{v})$ denotes the diagonal matrix with elements of vector $\mathbf{v}$ on the main diagonal. The first three components of block $\mathbf{M}_{r}$ are masses of the RFE, i.e. $m_{r, 1}=m_{r, 2}=m_{r, 3}=m_{r}$, the others are mass moments of inertia around axes $x_{r, 1}, x_{r, 2}, x_{r, 3}$.

Spring-damping elements are defined by blocks of stiffness and damping coefficients. These blocks are also diagonal, as $y_{k, 1}, y_{k, 2}, y_{k, 3}$ are main axes of the SDE. For spatial systems the block of stiffness coefficients for the $k$ th SDE has the following form [25]:

$$
\mathbf{K}_{k}=\operatorname{diag}\left(\left[\begin{array}{llllll}
k_{k, 1} & k_{k, 2} & k_{k, 3} & k_{k, 4} & k_{k, 5} & k_{k, 6}
\end{array}\right]\right) .
$$

The first three components of this block are translational stiffness coefficients along axes $y_{k, 1}, y_{k, 2}$, and $y_{k, 3}$, the others are rotational stiffness coefficients around the same axes.

The block of damping coefficients has a similar form [25]

$$
\mathbf{D}_{D, k}=\operatorname{diag}\left(\left[\begin{array}{llllll}
d_{k, 1} & d_{k, 2} & d_{k, 3} & d_{k, 4} & d_{k, 5} & d_{k, 6}
\end{array}\right]\right) .
$$

The position of each rigid finite element is defined with its general coordinates. For spatial systems the RFE has six degrees of freedom and consequently its position is defined with six general coordinates. These are, as follows: three displacements of its mass center along axes $x_{r, 1}, x_{r, 2}, x_{r, 3}$, and three rotations around the same axes. The six general coordinates form the following column vector [25]:

$$
\mathbf{q}_{r}=\left\{\begin{array}{llllll}
q_{r, 1} & q_{r, 2} & q_{r, 3} & q_{r, 4} & q_{r, 5} & q_{r, 6}
\end{array}\right]^{T} .
$$

Similarly, general forces loading the RFE along general displacements have the following form [25]:

$$
\mathbf{P}_{r}=\left\{\begin{array}{llllll}
P_{r, 1} & P_{r, 2} & P_{r, 3} & P_{r, 4} & P_{r, 5} & P_{r, 6}
\end{array}\right]^{T} \text {. }
$$

Blocks of inertia $\mathbf{M}_{r}$, of stiffness $\mathbf{K}_{k}$ and damping $\mathbf{D}_{k}$ coefficients are main components of global mass, damping and stiffness matrices of the whole structure (see Appendix A). The procedure of calculating the components of these blocks, as well as the procedure of constructing global matrices of the whole system are thoroughly explained in [25].

The original work of Kruszewski et al. [25] does not discuss the motion of rotating shafts. Only small displacements (vibrations) of non-rotating structures are considered. Hence, the equation of motion developed in [25] has to be modified in order to include gyroscopic forces and other effects characteristic for rotating shafts. Furthermore, the model of the crack has to be developed and introduced into the equation of motion. These problems will be presented and solved further, in the following sections.

\section{RFE model of the uncracked rotor}

\subsection{Equations of motion}

The proposed approach is demonstrated on a rotor supported by two ball bearings, consisting of a shaft and a disk. The rotor is a part of a crack detection test rig utilized at the Center for Rotating Machinery Dynamics and Control (RoMaDyC) at Cleveland State University [13]. Main dimensions and the schematic diagram of the rotor are presented in Fig. 2a.

Using the rigid finite element method [25], the rotor is divided into a selected number of lumped elements. The division runs in two steps.

In the first step, the rotor is divided into 50 elements (Fig. 2a). In the middle of each prismatic element, a springdamping element is located; in Fig. 2a SDEs are marked with crossed circles. Each of tapered elements, numbered as 3, 4, and 42,43 is replaced by 6 prismatic elements of equal length and gradually decreasing diameter, according to the procedure described in [25]. In the present case, spatial SDEs are selected, i.e. the elements that can be loaded with six general forces, of which three act along three different axes (translational forces) while the other three act around these 

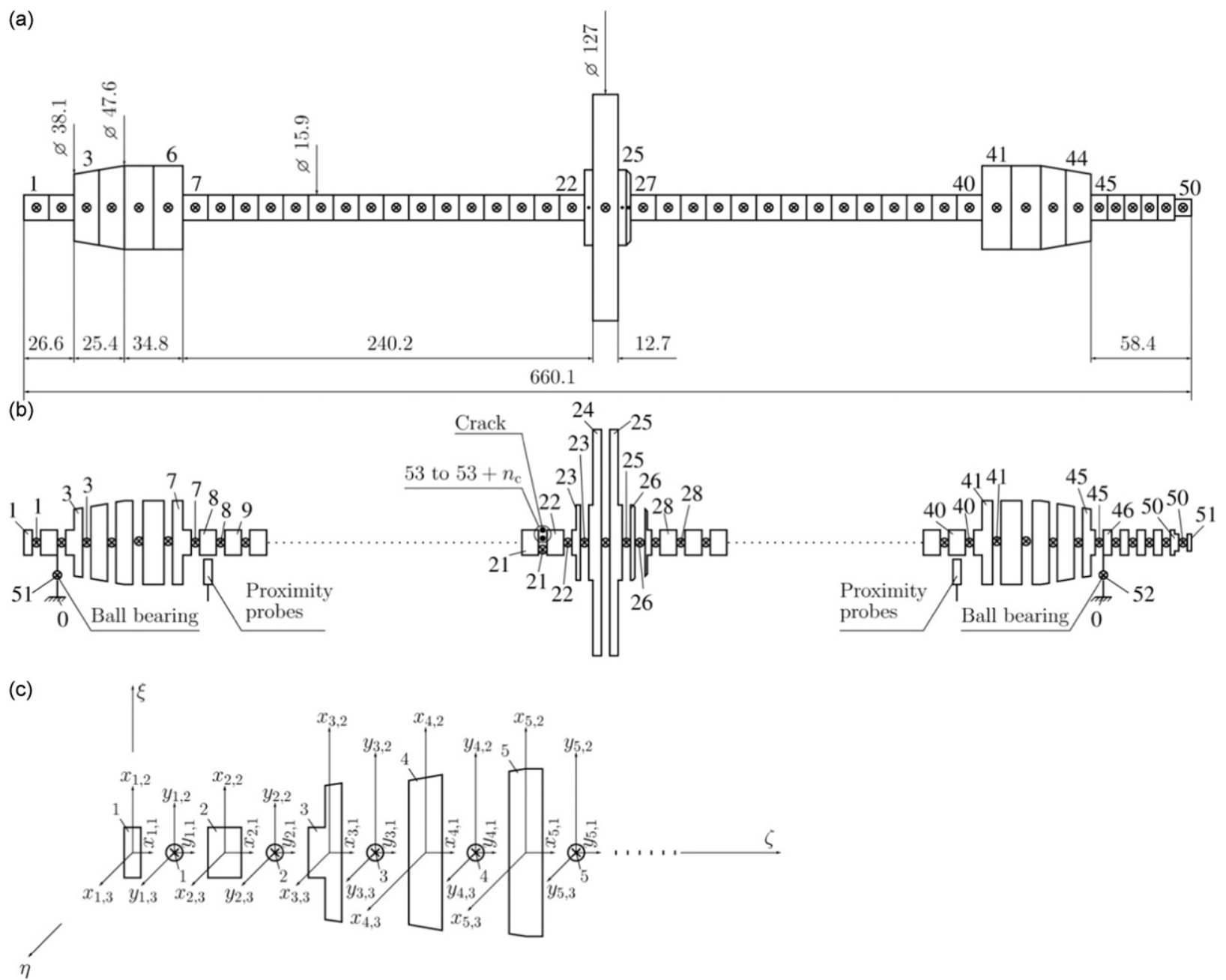

Fig. 2. Rigid finite element model of the rotor: (a) original division into 50 spring-damping elements, (b) secondary division into 51 rigid finite elements, (c) global coordinate system $\xi, \eta, \zeta$, and local coordinate systems of individual RFEs $\left(x_{r, 1}, x_{r, 2}, x_{r, 3}\right)$ and SDEs $\left(y_{k, 1}, y_{k, 2}, y_{k, 3}\right)$.

axes (torques). The motion of each SDE is considered in an independent coordinate system with its three main axes $y_{k, 1}$, $y_{k, 2}$ and $y_{k, 3}$ (Fig. 2c).

After dividing the rotor into spring-damping elements and after assuming their coordinate systems, the flexural and torsional stiffness coefficients along each six directions of each element are calculated according to the procedure described in [25].

In the second step, 51 rigid finite elements are located between the corresponding SDEs obtained in the first step. As in the first step, non-prismatic elements (e.g. 3, 4, 5, 7) are replaced by 6 prismatic RFEs, according to [25].

The motion of each of the RFEs is considered in an independent coordinate system with three main axes $x_{r, 1}, x_{r, 2}, x_{r, 3}$ (Fig. 2c). The center of the system is located at the mass center of a given element. At a standstill, axes $x_{r, 1}, x_{r, 2}$ and $x_{r, 3}$ coincide with three principal axes of the element; $x_{r, 1}$ coincides with the rotation axis.

Next, masses and mass moments of inertia of all 51 RFEs are calculated according to the procedure presented in [25].

Then, on the basis of stiffness coefficients, masses and mass moments of inertia of individual SDEs and RFEs, stiffness $K$ and mass $M$ matrices of the rotor are created [25].

Proportional damping is assumed with the damping matrix $\mathbf{D}_{D}$ calculated according to the known formulae [25,27]

$$
\mathbf{D}_{D}=\alpha_{D} \mathbf{M}+\beta_{D} \mathbf{K}
$$

where coefficients $\alpha_{D}$ and $\beta_{D}$ are assumed as follows: $\alpha_{D}=10^{-5}, \beta_{D}=0$.

The vibrations of the rotor are considered in a local coordinate system $\xi, \eta, \zeta$ which rotates with the rotor with its angular speed $\Omega$ around axis $\zeta$, which at a standstill is the rotor symmetry axis. Of course, coordinate systems of individual 
RFEs and SDEs rotate with the rotor, too. In the local coordinate system, the equations of motion take the following form:

$$
\mathbf{M} \ddot{\mathbf{q}}+\left(\mathbf{D}_{D}+\Omega \mathbf{D}_{G}\right) \dot{\mathbf{q}}+\mathbf{K q}=\mathbf{P},
$$

where $\mathbf{q}$ is a vector of displacements of the centers of masses of individual RFEs and $\mathbf{P}$ is a vector of forces (such as gravity, unbalance, etc.) loading the rotor. Matrix $\mathbf{D}_{G}$ includes gyroscopic effects, yet is not introduced in an original equation of motion, which has been developed in [25] in the following form:

$$
\mathbf{M} \ddot{\mathbf{q}}+\mathbf{D}_{D} \dot{\mathbf{q}}+\mathbf{K q}=\mathbf{P} .
$$

It is well justified, as only small displacements of non-rotating structures are considered in [25]. However, if the motion of the flexible rotating rotor is considered, then the gyroscopic effects must be absolutely included. Thus, the form of the gyroscopic matrix $\mathbf{D}_{G}$ for the rigid finite element, rotating around one of its principal axis and subjected to small transversal and/or rotational vibrations along/around the other axes should be determined. The simplified procedure of creating the gyroscopic matrix is presented in the following section.

\subsection{Gyroscopic matrix}

Fig. 3a presents the $r$ th rigid finite element rotating around axis $x_{r, 1}$ and subjected to small transversal and rotational deformations along/around axes $x_{r, 2}$ and $x_{r, 3}$. The motion of the element will be considered independently in two planes $x_{r, 1}, x_{r, 2}$ (Fig. 3b) and $x_{r, 1}, x_{r, 3}$ (Fig. 3c), in a local coordinate system $x_{r, 1}, x_{r, 2}, x_{r, 3}$ rotating with the element at constant speed $\Omega$.

Gyroscopic effects acting on the element will manifest themselves by two general forces:

(a) The Coriolis force $\mathbf{F}_{G, r}$ perpendicular to the vector of angular speed $\boldsymbol{\Omega}$ and resulting from translational velocities $\dot{q}_{r, 2}$, $\dot{q}_{r, 3}$ of the element lumped mass,

(b) Gyroscopic moment $\tau_{G, r}$ resulting from the rotations around axes $x_{r, 2}$ and $x_{r, 3}$ (with angular velocities $\dot{q}_{r, 6}$ and $\dot{q}_{r, 5}$ ) the element's lumped mass moments of inertia.

The general formulae for the Coriolis force is as follows:

$$
\mathbf{F}_{G, r}=-2 m_{r} \boldsymbol{\Omega} \times \mathbf{v}_{r}
$$

while for the gyroscopic moment

$$
\boldsymbol{\tau}_{G, r}=J_{r, 1} \omega_{r} \times \mathbf{\Omega},
$$

where $m_{r}, J_{r, 1}$ are the element's lumped mass and lumped mass moment of inertia (around the rotation axis $x_{r, 1}$ ), $\mathbf{v}_{r}$ is the

(a)

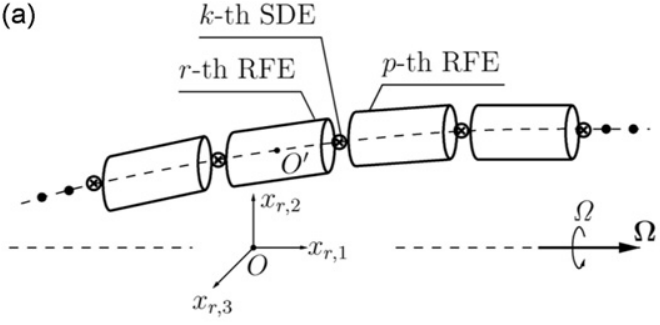

(b)

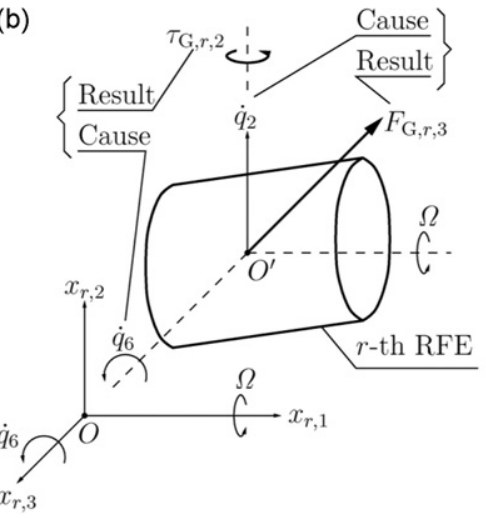

(c)

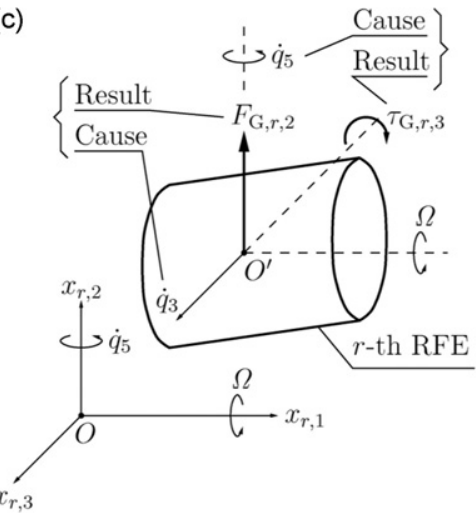

Fig. 3. Rigid finite element of a rotating shaft: (a) schematic of the shaft, (b) motion in plane $x_{r, 1}, x_{r, 2}$, and (c) motion in plane $x_{r, 1}, x_{r, 3}$. 
vector of element's translational velocity, $\omega_{r}$ is the vector of element's angular speed, where

$$
\mathbf{v}_{r}=\left\{\begin{array}{lll}
\dot{q}_{r, 1} & \dot{q}_{r, 2} & \dot{q}_{r, 3}
\end{array}\right]^{T}, \quad \boldsymbol{\omega}_{r}=\left[\begin{array}{lll}
\dot{q}_{r, 4} & \dot{q}_{r, 5} & \dot{q}_{r, 6}
\end{array}\right]^{T} .
$$

From the above, for the motion in plane $x_{r, 1}, x_{r, 2}$, the Coriolis force acting along axis $x_{r, 3}$ will be

$$
F_{G, r, 3}=-2 m_{r} \Omega \dot{q}_{r, 2},
$$

while the gyroscopic moment around axis $x_{r, 2}$

$$
\tau_{G, r, 2}=J_{r, 1} \Omega \dot{q}_{r, 6} .
$$

Similarly, for the motion in plane $x_{r, 1}, x_{r, 3}$, the Coriolis force along axis $x_{r, 2}$ will be

$$
F_{G, r, 2}=2 m_{r} \Omega \dot{q}_{r, 3}
$$

and the gyroscopic moment around axis $x_{r, 3}$

$$
\tau_{G, r, 3}=-J_{r, 1} \Omega \dot{q}_{r, 5} .
$$

From Eqs. (12)-(15) the general vector $\mathbf{P}_{G, r}$ of Coriolis forces and gyroscopic moments can be calculated as

$$
\mathbf{P}_{G, r}=-\Omega \mathbf{D}_{G, r} \dot{\mathbf{q}}_{r}
$$

where

and the gyroscopic matrix $\mathbf{D}_{G, r}$ takes the following form:

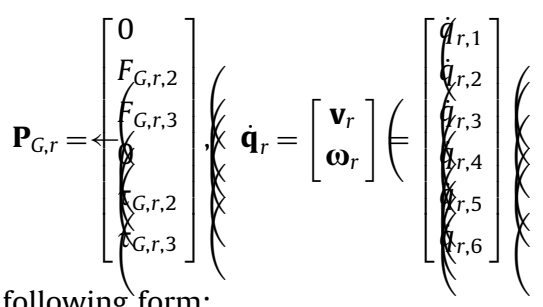

$$
\mathbf{D}_{G, r}=\left[\begin{array}{cccccc}
\emptyset & 0 & 0 & 0 & 0 & 0 \\
\oint & 0 & -2 m_{r} & 0 & 0 & 0 \\
\hat{\oint} & 2 m_{r} & 0 & 0 & 0 & 0 \\
\oint & 0 & 0 & 0 & 0 & 0 \\
\oint & 0 & 0 & 0 & 0 & -J_{r, 1} \\
( & 0 & 0 & 0 & J_{r, 1} & 0
\end{array}\right]
$$

Introducing Eq. (16) into the equation of motion (8) adapted for the $r$ th RFE, yields

$$
\mathbf{M}_{r} \ddot{\mathbf{q}}_{r}+\hat{\mathbf{D}}_{D, r} \dot{\mathbf{q}}+\hat{\mathbf{K}}_{r} \mathbf{q}=\mathbf{P}_{G, r}+\mathbf{P}_{r}
$$

or after some rearrangements

$$
\mathbf{M}_{r} \ddot{\mathbf{q}}_{r}+\hat{\mathbf{D}}_{D, r} \dot{\mathbf{q}}+\Omega \mathbf{D}_{G, r} \dot{\mathbf{q}}_{r}+\hat{\mathbf{K}}_{r} \mathbf{q}=\mathbf{P}_{r}
$$

In Eqs. (19) and (20) the $6 \times 6 n_{r}$ matrices $\hat{\mathbf{K}}_{r}$ and $\hat{\mathbf{D}}_{D, r}$ denote the $r$ th row stripe of the stiffness $\mathbf{K}$ and damping $\mathbf{D}_{D}$ matrices.

Eq. (20) results in Eq. (7) if all $n_{r}$ RFEs are considered. Gyroscopic matrix $\mathbf{D}_{G}$ for the whole rotor system is created from gyroscopic matrices $\mathbf{D}_{G, r}$ of individual RFEs (using the procedure similar to the one applied for the general mass matrix [25]) and then introduced into Eq. (7). The form of matrix $\mathbf{D}_{G}$ is presented in Appendix A.

\subsection{Model verification}

On the basis of the general matrices $\mathbf{M}, \mathbf{D}_{D}, \mathbf{D}_{G}, \mathbf{K}$ of the rotor, its dynamic properties may be evaluated. Fig. 4 presents the transfer function of the non-rotating, free-free rotor (no bearings) obtained experimentally (continuous line) and from the RFE model (dashed line). The experimental transfer function has been measured using the impact hammer modal testing. The rotor was suspended in the air using thin nylon wire and struck with the force-instrumented hammer. The resultant motion of the rotor was measured with an accelerometer fixed on the rotor and then analyzed using the dynamic signal analyzer. The modeled transfer function has been obtained using the Bode plot.

A very good agreement can be observed between the modeled and experimental data. The values of first three natural frequencies, which for the experiment are $84.25 \mathrm{~Hz}, 362 \mathrm{~Hz}$ and $646 \mathrm{~Hz}$, agree very well with the values obtained from the RFE model $(84 \mathrm{~Hz}, 356.62 \mathrm{~Hz}$ and $642 \mathrm{~Hz}$ respectively). The agreement between their amplitudes is not so good, which can be explained by improper estimation of the proportional damping matrix $\mathbf{D}_{D}$. From the experimental data, one can conclude that a more accurate idea may include the use of the modal damping matrix, where the damping coefficients for 


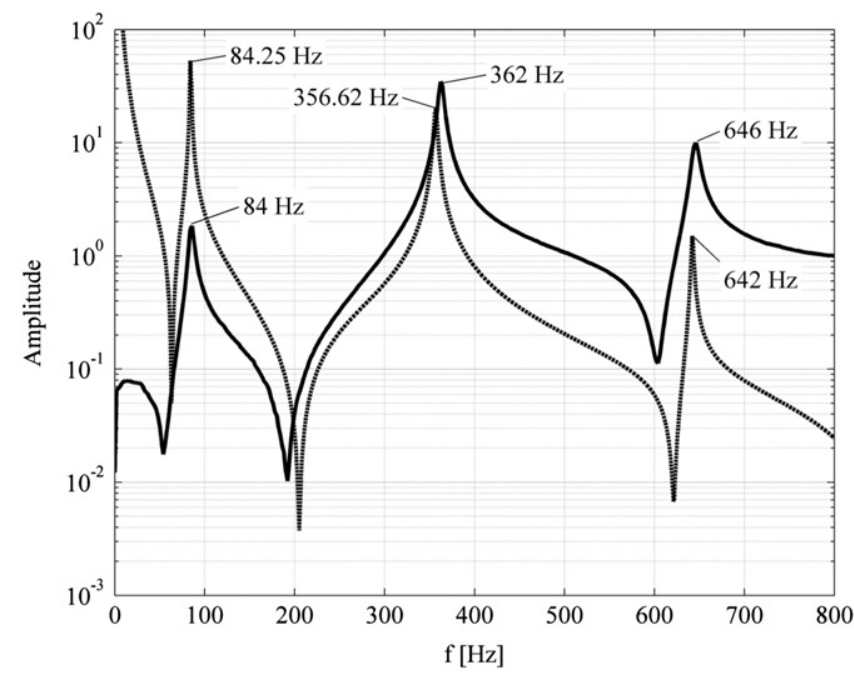

Fig. 4. Transfer function of the free-free rotor (continuous line) and its RFE model (dashed line).

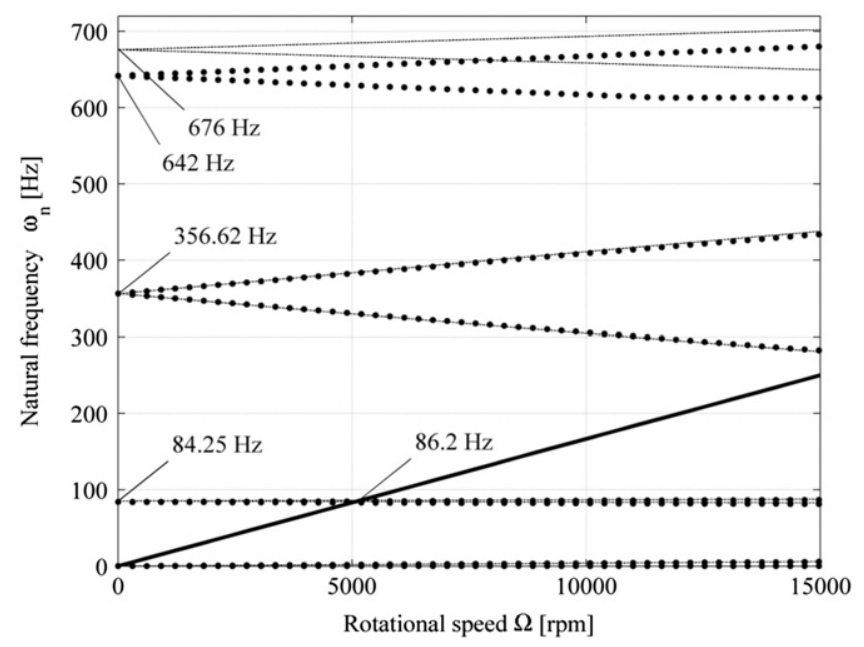

Fig. 5. Campbell diagram of the RFE (dots) and XLRotor (dashed lines) models of the free-free rotor; only gyroscopic moment included.

first three natural frequencies are chosen individually. Certainly, it is possible to use modal damping when the RFE approach is applied, however it has not been applied for the present study.

Fig. 5 shows the Campbell diagram of the free-free rotor. Dotted lines have been calculated using the RFE model. For comparison, the dashed lines have been calculated using the commercial finite element software XLRotor [28]. An almost perfect agreement between these two result sets can be noticed, especially for the first two natural frequencies $(84.25 \mathrm{~Hz}$ and $356.62 \mathrm{~Hz}$ ) and their changes with rotor rotational speed. Although the situation for the third frequency is less agreeable (642 Hz obtained from RFE and 676 obtained from XLRotor), the veering of this frequency with the rotational speed are still similar.

\section{RFE model of the cracked rotor}

\subsection{Model of the crack}

The concept of the proposed rigid finite element model of the transverse shaft crack will be presented in this section.

Fig. 6 shows two rigid finite elements and the cross section of the shaft at the location of the crack. The crack edge line located at depth $a$ from the side surface of the shaft separates the uncracked (hatched) and cracked areas.

The crack is supposed to be located between the two RFE elements (numbered as $r$ and $p$ ) and is modeled using several spring-damping elements connecting these RFEs. To simplify the considerations, it is assumed that the main SDE connecting the two RFEs (marked with a large crossed circle in Fig. 6(b) and representing the stiffness of the uncracked 
(a)

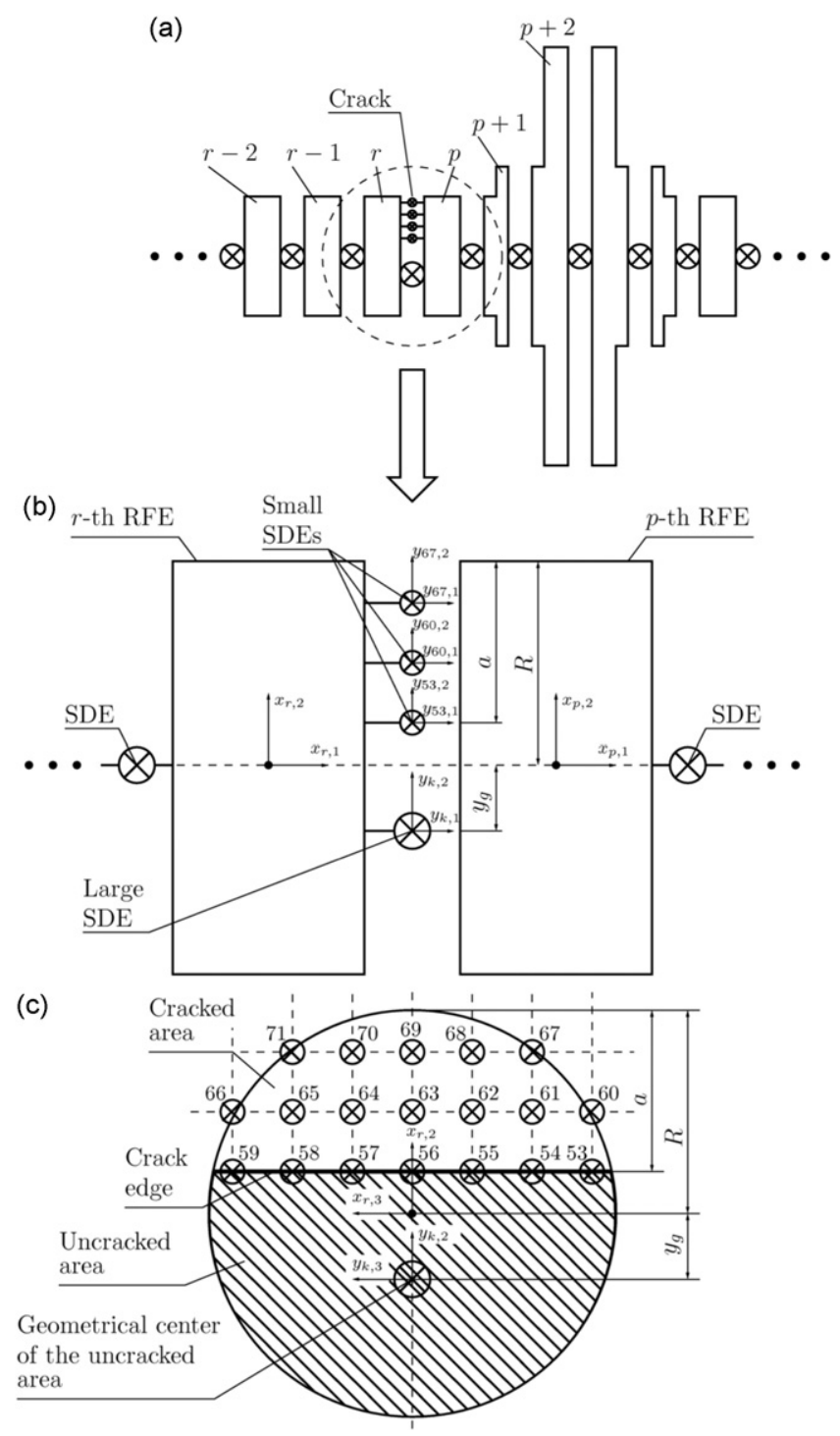

Fig. 6. Model of the crack: (a) possible location of the crack, (b) two RFEs and several SDEs at the location of the crack, and (c) shaft cross section at the location of the crack.

area) is located at the geometrical center of the uncracked area. Furthermore, the stiffness of this SDE is proportional to the crack depth. In fact such dependency is nonlinear, as the stiffness depends on the cross sectional area and on the area moment of inertia of the uncracked segment. To improve the accuracy, the more exact assumptions can be taken, as explained in Appendix C. For the details the reader is referenced to [6,29].

Nevertheless, for the rest of the paper it is assumed that the stiffness coefficients of the large SDE in main six directions are constant and proportional to the relative depth of the uncracked area $(1-\mu)$. Here, $\mu$ is the relative depth of the crack and is defined as follows:

$$
\mu=\frac{a}{2 R}
$$

where $R$ is the radius of the shaft. This way stiffness coefficients of the large SDE are evaluated as

$$
k_{C, k, j}=(1-\mu) k_{U, k, j}
$$

where $k_{U, k j}$ are stiffness coefficients of the SDE, which would connect the two RFEs in case of the uncracked shaft, $j=1,2, \ldots, 6$. Stiffness coefficients $k_{U, k j}$ are determined using the procedure described in [25].

The cracked area between the two RFEs is connected with several smaller SDEs (there are 19 SDEs in Fig. 6c numbered from 53 to 71). These SDEs are located at selected points of the uncracked area, e.g. at the nodes of the imaginary geometrical array, formed with vertical and horizontal lines running perpendicularly and parallel to the crack edge. 
Stiffness coefficients $k_{k, j}$ of the individual SDEs are equal and are chosen in such a way that their sums in all six directions are proportional to the relative depth of the crack $\mu$, i.e.

$$
\sum_{k=53}^{53+n_{c}}\left(k_{k, j}=\mu k_{U, k, j}\right.
$$

where $n_{c}$ is the number of small SDEs, $j=1,2, \ldots, 6$. This way the sum of the stiffness coefficients of small SDEs $k_{k, j}$ and a large SDE $k_{C, k, j}$ in a particular direction $j$ equals the stiffness of the uncracked SDE $k_{U, k, j}$

$$
k_{C, k, j}+\sum_{k=5,3}^{53+n_{c}}\left(k_{k, j}=k_{U, k, j} .\right.
$$

The situation described above illustrates the fully closed crack state. If crack breathing is to be accounted for, then the stiffness of small SDEs should change. In practice, these changes are determined in a way described below.

The stiffness of an individual small SDE in a given direction $j$ can be zero or $k_{k, j}$ depending on the deformation $\Delta w_{k, 1}$ of this SDE along axis $y_{k, 1}$ (i.e. for $j=1$ ). If this deformation is greater than zero, this means that the corresponding SDE is compressed, its stiffness should be $k_{k, j}$ and the crack at its location is closed. It can be vividly presented, as if the springdamper touches the crack surface (Fig. 7). On the other hand if this deformation is less or equal to zero, then the corresponding SDE is under tension, its stiffness should be zero and the crack at its location is opened. It can be presented as if the spring-damper does not touch the surface of the crack. This way, based on the sign of the deformation of all small SDEs in direction perpendicular to the crack surface, the open/close state of the crack can be easily determined, and the corresponding stiffness change can be introduced into the stiffness matrices. Fig. 7 shows two situations: the first, when a single small SDE is compressed (closed crack), and the second, when the small SDE is under tension (open crack).

Deformations of a given spring-damping element are expressed with vector $\Delta \mathbf{w}_{k}$ composed of six components: the first three are translational deformations along corresponding axes $y_{k, 1}, y_{k, 2}, y_{k, 3}$, and the next three are rotational deformations around the same axes. According to [25], vector $\Delta \mathbf{w}_{k}$ can be calculated, as

$$
\Delta \mathbf{w}_{k}=\mathbf{T}_{r, k} \mathbf{q}_{r}-\mathbf{T}_{p, k} \mathbf{q}_{p},
$$

where $\mathbf{q}_{r}$ and $\mathbf{q}_{p}$ are vectors of displacements of the $r$ th and $p$ th RFE connected with the $k$ th SDE, and $\mathbf{T}_{r, k}$ and $\mathbf{T}_{p, k}$ are corresponding transformation matrices [25].

Calculating the vibration response of the cracked rotor, the procedure of determining the crack open/close state is performed according to following steps:

1. Initially, the crack is supposed to be completely opened, i.e. stiffness matrices of all small SDEs are zero and only the stiffness matrix of the large SDE is introduced into the stiffness matrix $\mathbf{K}$ of the whole system (Eq. (7)).

2. For the given time step, the response vector $\mathbf{q}$ is calculated according to Eq. (7) and vectors of displacements $\mathbf{q}_{r}$ and $\mathbf{q}_{p}$ of the two RFEs between the crack are excluded from it.

3. Deformation vectors $\Delta \mathbf{w}_{k}$ of all small SDEs are calculated, according to Eq. (25).

4. If the first component $\Delta w_{k, 1}$ of the given vector $\Delta \mathbf{w}_{k}$ is less or equal to zero, then the corresponding SDE is opened and its stiffness matrix remains zero. Otherwise, the corresponding SDE is closed and its stiffness matrix is modified to contain $k_{k, j}$ components.

5. Stiffness matrix $\mathbf{K}$ of the whole system is updated with modified matrices of all small SDEs and the procedure from 2 to 5 is repeated with the new value of $\mathbf{K}$.

(a)

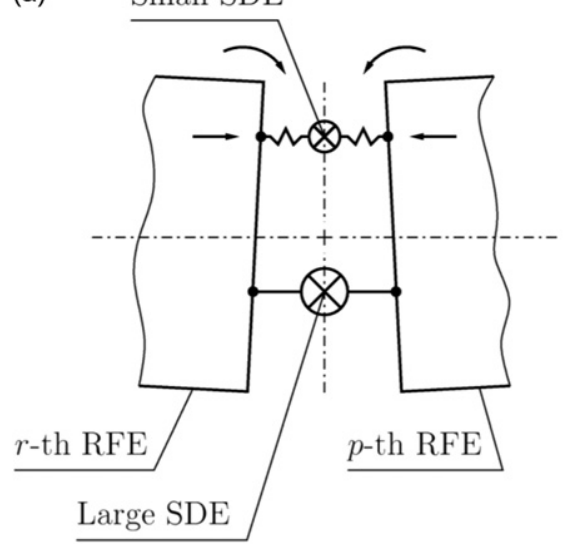

(b)

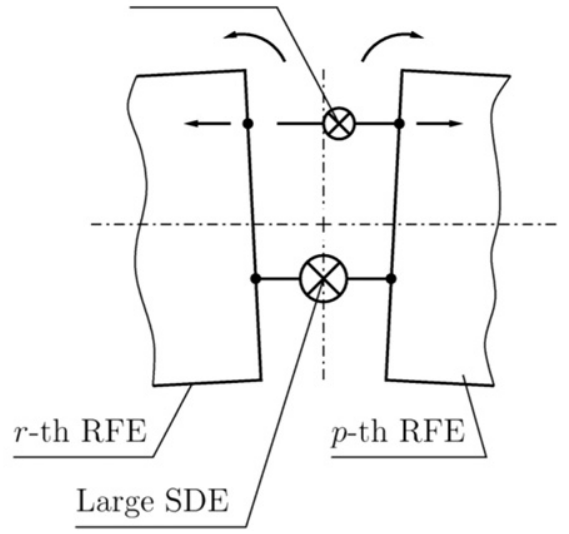

Fig. 7. Possible deformations of the small SDE: (a) compression and (b) tension. 
In practical calculations, the number of small SDEs between the crack lips should be chosen in a way to ensure the realistic behavior of the crack breathing. This is not a problem in case of RFE method, where increasing the number of SDEs is straightforward and is performed in the same systematic way (the only difficulty is to presumably calculate the coordinates of the pinning points of all small SDEs on the cracked surface).

The proposed model allows one to analyze also other mechanisms that may occur at the crack location. If the procedure described above detects the compressed state of the individual SDE, then its stiffness matrix can be modified in such a way that only stiffness changes in selected directions (e.g. stiffnesses for compression, shearing, bending or torsion) can be accounted for. Shearing, sliding or torsional effects between crack lips can be easily investigated this way.

\subsection{Model of the cracked rotor}

The rigid finite element model of the cracked rotor described in Section 3.1 is created by including two supporting ball bearings and the model of the crack.

The bearings are modeled using two additional spring-damping elements connecting the basis (numbered as 0 ) with the 2nd and with the 46th RFE (Fig. 2b). The values of the radial bearing's stiffness $k_{B}$ and damping $d_{B}$ are assumed, as $k_{B}=3.6 \times 10^{6} \mathrm{~N} / \mathrm{m}$, and $d_{B}=10 \mathrm{~N} \mathrm{~s} / \mathrm{m}$, and introduced into the corresponding stiffness and damping matrices of the two SDEs.

The crack is located between the 21st and the 22nd RFE (Fig. 2b), and is modeled using one large SDE and several hundred small SDEs. Small SDEs are distributed evenly at the nodes of the rectangular mesh mapped on the cracked area of the crack cross section. The rotor response is calculated including the breathing mechanism described in Section 4.1. Two different cases of small SDEs stiffness changes have been considered: the first, when during the breathing, the stiffness of each small SDE may change in all six directions, and the second, when only changes in translational stiffness along axis $y_{k, 1}$ and in torsional stiffness around axes $y_{k, 2}$ and $y_{k, 3}$ perpendicular to the shaft axis are allowed (Fig. 2c). In the latter case the translational stiffness along axes $y_{k, 2}$ and $y_{k, 3}$ and the torsional stiffness around axis $y_{k, 1}$ are all time zero, what corresponds to the hypothetical situation when the lips of the crack slide on each other with almost no friction. The first case will be referenced to as the full stiffness, while the second as the half stiffness change.

The calculations have been conducted for two different cracks: the first of the 25 percent, and the second of the 40 percent relative depth. In the first case the number of small SDEs connecting the $21 \mathrm{st}$ with the $22 \mathrm{nd}$ RFE is $n_{c}=260$, while in the second case it is $n_{c}=488$.

According to Eq. (7), the response $q$ of the rotor is calculated using the rotating reference frame $\xi, \eta$, $\zeta$ and then is transformed to the inertial frame $x, y, z$ (Fig. 8) using the following formulae:

$$
\mathbf{q}_{g}=\mathbf{T}_{g} \mathbf{q}
$$

where for the instantaneous time $t$ the diagonal transformation matrix $\mathbf{T}_{g}$ is assembled using the elemental coordinate transformation matrix $\mathbf{T}_{e}$, given by

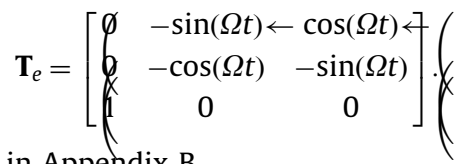

The derivation of matrix $\mathbf{T}_{e}$ is presented in Appendix B.

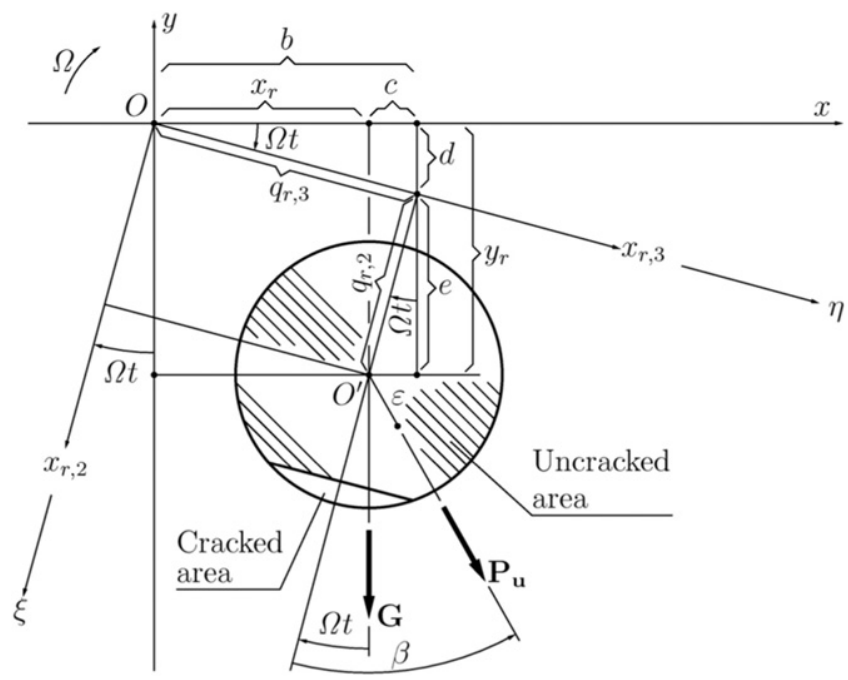

Fig. 8. Rotor crack section in inertial and rotating coordinates. 
Vector $\mathbf{P}$ of forces loading the rotor is composed of gravity $\mathbf{G}$ and unbalance $\mathbf{P}_{u}$

$$
\mathbf{P}=\mathbf{G}+\mathbf{P}_{u}
$$

In the rotating coordinate system the unbalance is constant, and its components in directions $x_{r, 2}$ and $x_{r, 3}$ are, as follows:

$$
P_{u, r, 2}=m_{r} \varepsilon \Omega^{2} \cos (\beta), \quad P_{u, r, 3}=m_{r} \varepsilon \Omega^{2} \sin (\beta),
$$

where $\beta$ is the angle between the crack and unbalance vector and $\varepsilon$ is the eccentricity of rotor elements. The calculations have been conducted for two values of $\beta\left(\beta=45^{\circ}\right.$ and $\left.180^{\circ}\right)$ and two values of eccentricity $\left(\varepsilon=10^{-3} \mathrm{~m}\right.$ and $\left.10^{-4} \mathrm{~m}\right)$.

On the other hand, vector of gravity $\mathbf{G}$ rotates around the axis of the rotor with its angular speed $\Omega$ and its components in the local coordinate system are, as follows:

$$
G_{r, 2}=m_{r} g \cos (\Omega t), \quad G_{r, 3}=m_{r} g \sin (\Omega t),
$$

where $g$ is gravity acceleration.

Equation of motion (Eq. (7)) is solved using Newmark integration scheme [30], as it turned out to be more efficient for the analyzed rotor. All calculations are conducted for the rotor rotating with the angular speed of $\Omega=27 \mathrm{~Hz}$ ( $1620 \mathrm{rev} / \mathrm{min}$ ) till the 1000th revolution is obtained. The results present translational and angular displacements of the rotor at the selected point located at the mass center of the 8th rigid finite element (Fig. 2b), which is the location of the vibration probe.

\subsection{Dynamic properties of the uncracked rotor supported by bearings}

Natural frequencies of the uncracked rotor supported by ball bearings are different than those for the free-free rotor (see Section 3.3). This is due to additional stiffness and damping introduced by bearings. Fig. 9 presents the Campbell diagram for the uncracked rotor supported by ball bearings. The first two natural frequencies are shifted to $45 \mathrm{~Hz}$ and $225 \mathrm{~Hz}$ (compare to Fig. 5). Backward and forward critical speeds located at $44.9 \mathrm{~Hz}$ and $45.1 \mathrm{~Hz}$ are also shown.

Fig. 10 shows the response of the uncracked rotor loaded with unbalance and gravity. As expected the orbit is almost perfectly circular (Fig. 10b) and the distinct component of the synchronous frequency of $27 \mathrm{~Hz}$ (marked as $1 \times$ ) is present at the vertical vibration spectrum (Fig. 10c).

\subsection{Results for the 25 percent cracked rotor}

Fig. 11 presents the breathing of the 25 percent cracked rotor during the 1000 th revolution in the local frame. The small open circle denotes the direction of the gravity vector, while the asterisk - the direction of the unbalance $\left(\beta=45^{\circ}\right.$ in this case). Compressed small SDEs are marked with small black dots, delimiting the closed part of the crack from the uncracked one.

It can be noticed that the crack breathes in a completely different manner than this suggested by Papadopoulos and Dimarogonas [19], Darpe et al. [10], and others. The line delimiting the closed part of the crack from the uncracked one is not all time perpendicular to the crack edge. It rather rotates around the center of the shaft in a way similar to the motion of a clock hand. This behavior corresponds well with the results obtained by Bachschmid et al. [21], yet the present approach is simpler.

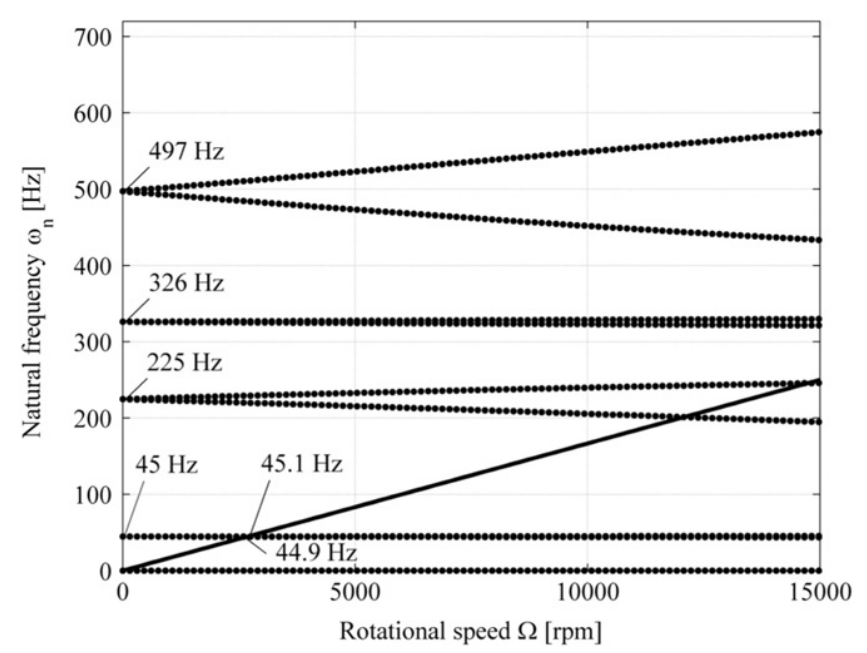

Fig. 9. Campbell diagram of the RFE model of the uncracked rotor supported by ball bearings; only gyroscopic moment included in the gyroscopic matrix. 

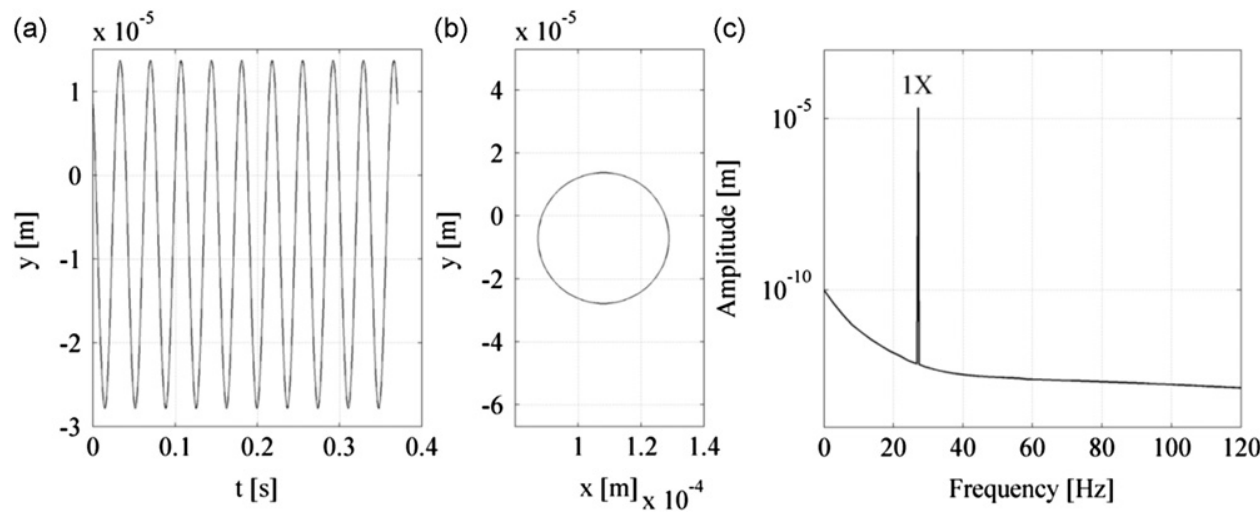

Fig. 10. Response of the uncracked rotor: (a) vertical vibration, (b) orbit and (c) vertical vibration spectrum; $\varepsilon=10^{-4} \mathrm{~m}$.

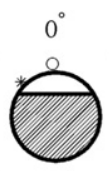

$90^{\circ}$

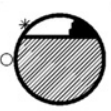

$180^{\circ}$
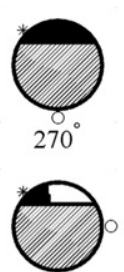

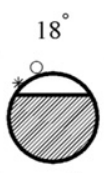

$108^{\circ}$

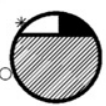

198

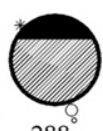

288

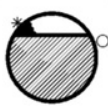

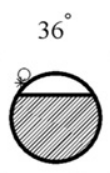
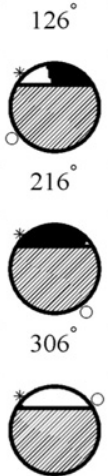

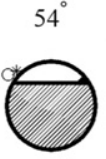

$144^{\circ}$
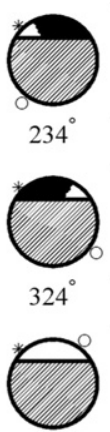
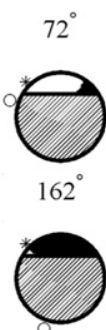

$252^{\circ}$
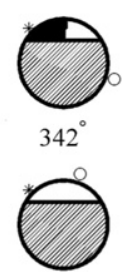

Fig. 11. Crack breathing: 25 percent crack, $\beta=45^{\circ}, \varepsilon=10^{-4} \mathrm{~m}$, full stiffness change.

The function graph of the crack closing is presented in Fig. 12d, where the crack closing $c_{c}$ is defined as the percentage ratio between the number of small SDEs under compression $n_{c c}$ to the total number $n_{c}$ of all small SDEs connecting the cracked part of the shaft

$$
c_{c}=\frac{n_{c c}}{n_{c}} \times 100 \text { percent }
$$

The change in this parameter during one revolution can be interpreted as the well known crack steering function, reported in $[5,6,14]$, and others.

Fig. 12 contains also the time response, the orbit and the frequency spectrum of the 25 percent cracked rotor. Frequency spectrum consists mainly of the synchronous frequency of $27 \mathrm{~Hz}(1 \times)$ and its harmonics $(2 \times, 3 \times, 4 \times)$, what is characteristic for the cracked shafts. However, small components of $18 \mathrm{~Hz}$ and $72 \mathrm{~Hz}$ can also be observed. In practical applications, these components could be hardly noticed, as their amplitudes are close to precision limits of modern measuring instruments, yet the appearance of these components should be explained.

Sawicki et al. [12], derived an important formula that can be used to explain the appearance of different harmonics in the vibration spectra of cracked, nonlinear rotors. He employed a harmonic balance analysis of the finite element model of the cracked rotor excited by an external harmonic force perpendicular to the shaft. The form of this formula is, as follows:

$$
\omega_{i}=r \Omega+s \Omega_{2} \text {, for some } i, r \text { and } s,
$$

where $\omega_{i}$ is the $i$ th natural frequency, $\Omega_{2}$ is the frequency of the excitation force and $\Omega$ is the rotor spin speed.

Eq. (31) gives the condition needed for appearing the so called combination resonances between the rotor angular speed $\Omega$, its natural frequencies $\omega_{i}$ and the excitation frequency $\Omega_{2}$. For given $\Omega$ and $\omega_{i}$ the frequencies of combination resonances can be calculated from Eq. (31) as subsequent $\Omega_{2}$. 
(a)

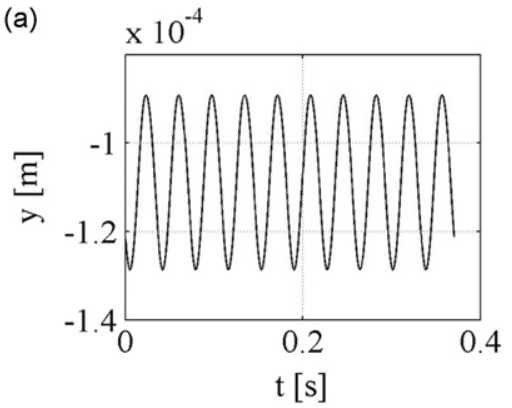

(c)

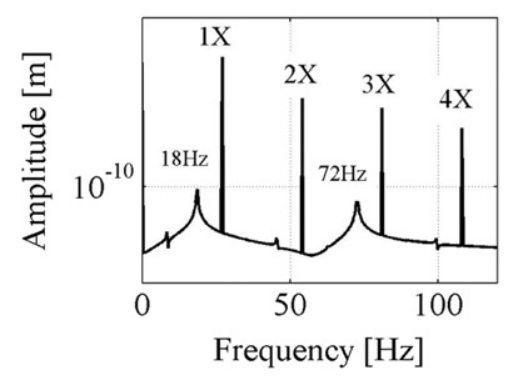

(b)

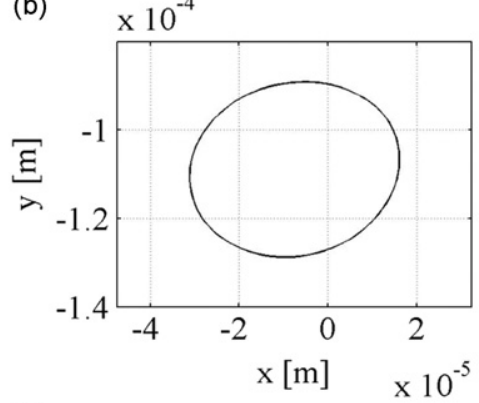

(d)

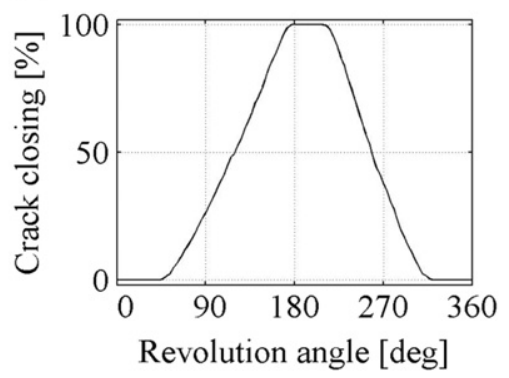

Fig. 12. Cracked rotor response: (a) vertical vibration, (b) orbit, (c) frequency spectrum and (d) crack closing; 25 percent crack, $\beta=45^{\circ}, \varepsilon=10^{-4} \mathrm{~m}$, full stiffness change.
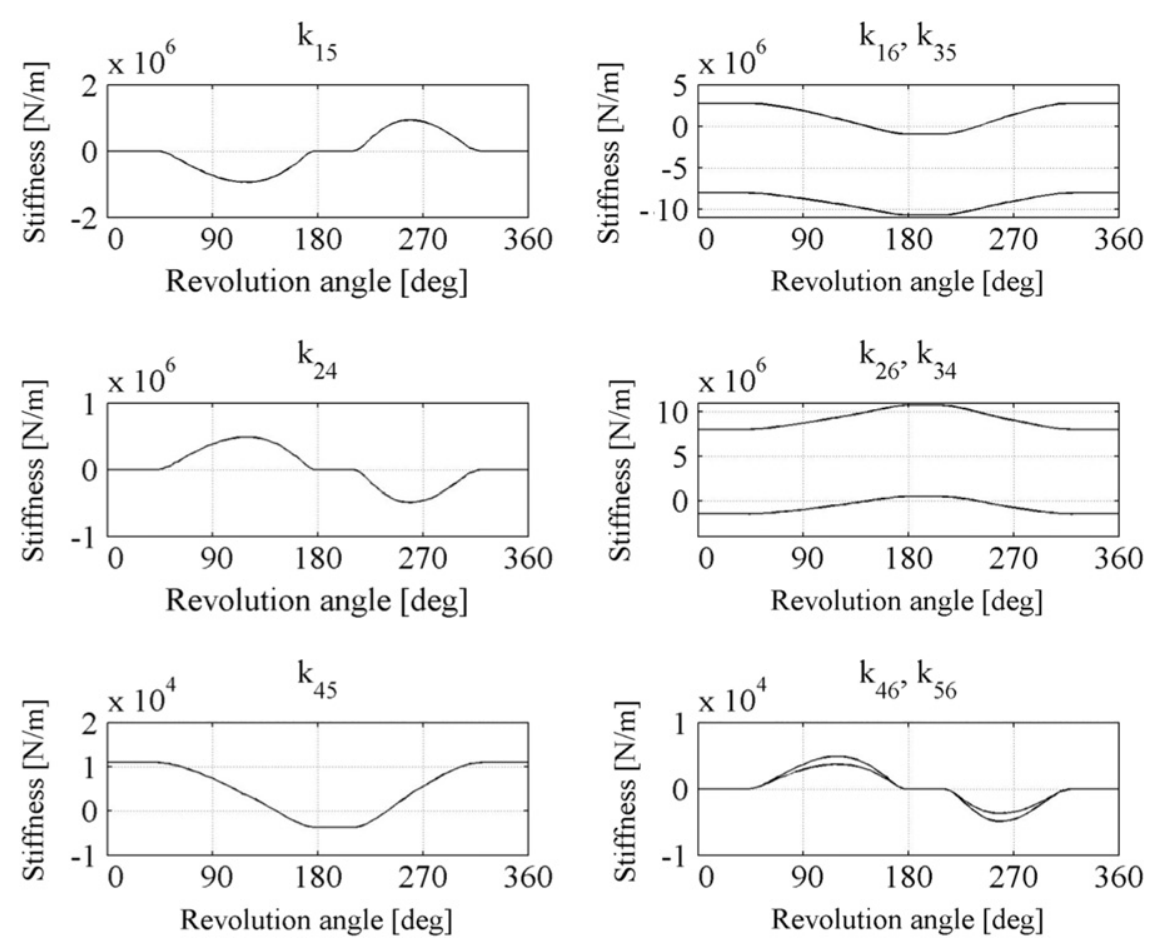

Fig. 13. Cracked area stiffness change: 25 percent crack, $\beta=45^{\circ}, \varepsilon=10^{-4} \mathrm{~m}$, full stiffness change.

For the analyzed rotor, introducing $\Omega=27 \mathrm{~Hz}, \omega_{1}=45 \mathrm{~Hz}, i=1, s=1$, and $r=1$ into Eq. (31) results in $\Omega_{2}=18 \mathrm{~Hz}$. Similarly, introducing $\Omega=27 \mathrm{~Hz}, \omega_{i}=45 \mathrm{~Hz}, i=1, s=-2$ and $r=7$ yields $\Omega_{2}=72 \mathrm{~Hz}$. These simple calculations explain the appearance of weak frequency peaks located at $18 \mathrm{~Hz}$ and $72 \mathrm{~Hz}$.

Fig. 13 shows changes in stiffness coefficients during one revolution. These coefficients are the components of the $6 \times 6$ matrix $\mathbf{K}_{21,21}^{*}$ located at the crossing of the 21 st row and the 21 st column stripes of the stiffness matrix $\mathbf{K}$. The changes in 
matrix $\mathbf{K}_{21,21}^{*}$ illustrate the stiffness changes of the shaft at the location of the crack during the breathing. Only the crosscoupled stiffness coefficients $\left(k_{15}, k_{16}, k_{24}, k_{26}, k_{34}, k_{35}, k_{45}, k_{46}, k_{56}\right)$ are shown, as the direct ones $\left(k_{11}, k_{22}, k_{33}, k_{44}, k_{55}\right.$, and $\left.k_{66}\right)$ increase to a maximum when the crack fully closes. The values of all coefficients change in a way corresponding to the breathing behavior shown in Figs. 11 and 12(d), as the full stiffness change has been assumed. It is worth noting that the results presented in Fig. 13 correspond very well with the ones obtained by Darpe et al. [10].

If the half stiffness change is assumed, then some cross-coupled stiffness coefficients remain zero during the whole revolution of the rotor. These are: $k_{24}, k_{26}, k_{34}, k_{35}, k_{45}, k_{46}$. This is presented in Fig. 14 . However, zero values of these coefficients do not result in a noticeable change of the rotor behavior, as for the half stiffness change, the breathing, the time response and the frequency spectrum of the vertical vibration are almost the same, as those presented in Figs. 11 and 12 for the full stiffness change. Furthermore, axial and torsional vibrations, coupled with the lateral ones are also not influenced by the changes in friction between the lips of the crack. It can be seen in Fig. 15 where the frequency spectra for axial and torsional
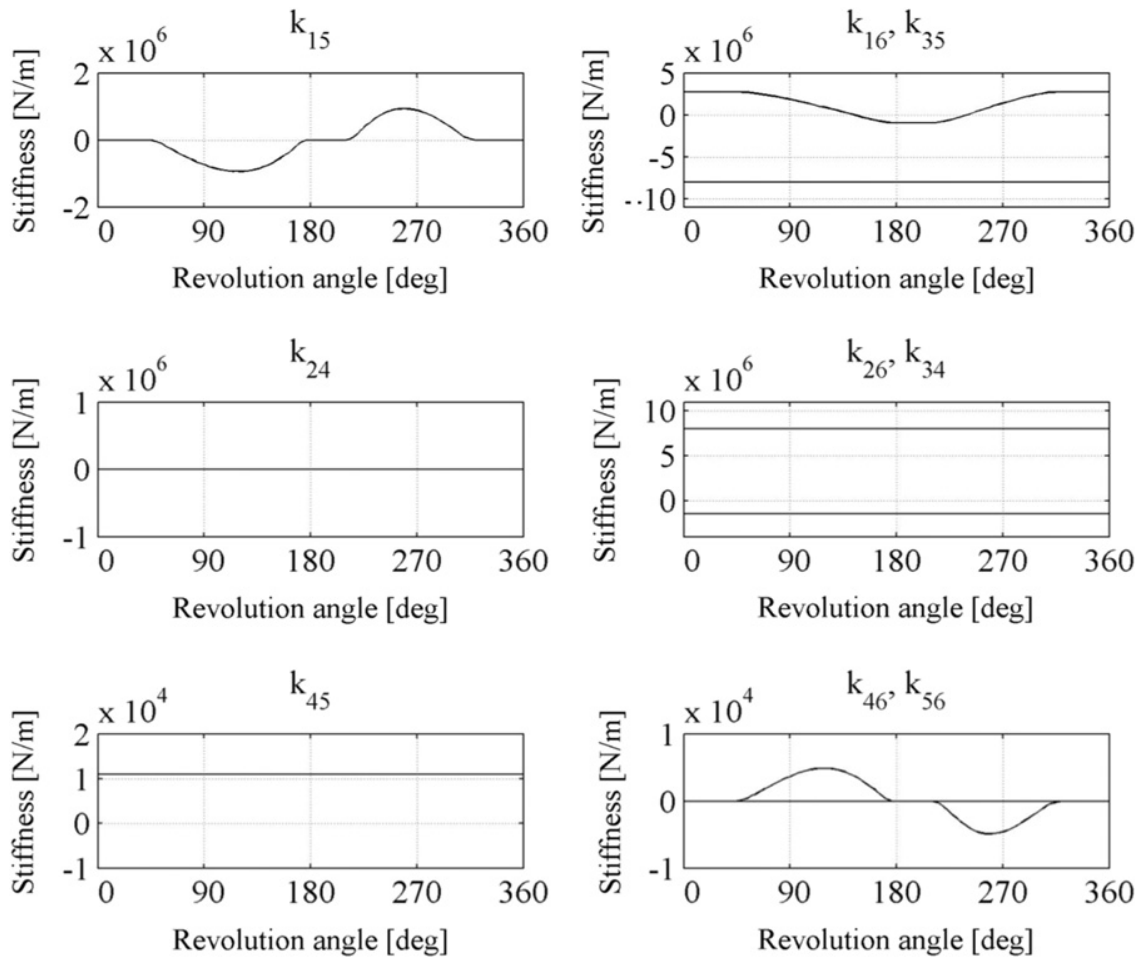

Fig. 14. Cracked area stiffness change: 25 percent crack, $\beta=45^{\circ}, \varepsilon=10^{-4} \mathrm{~m}$, half stiffness change.
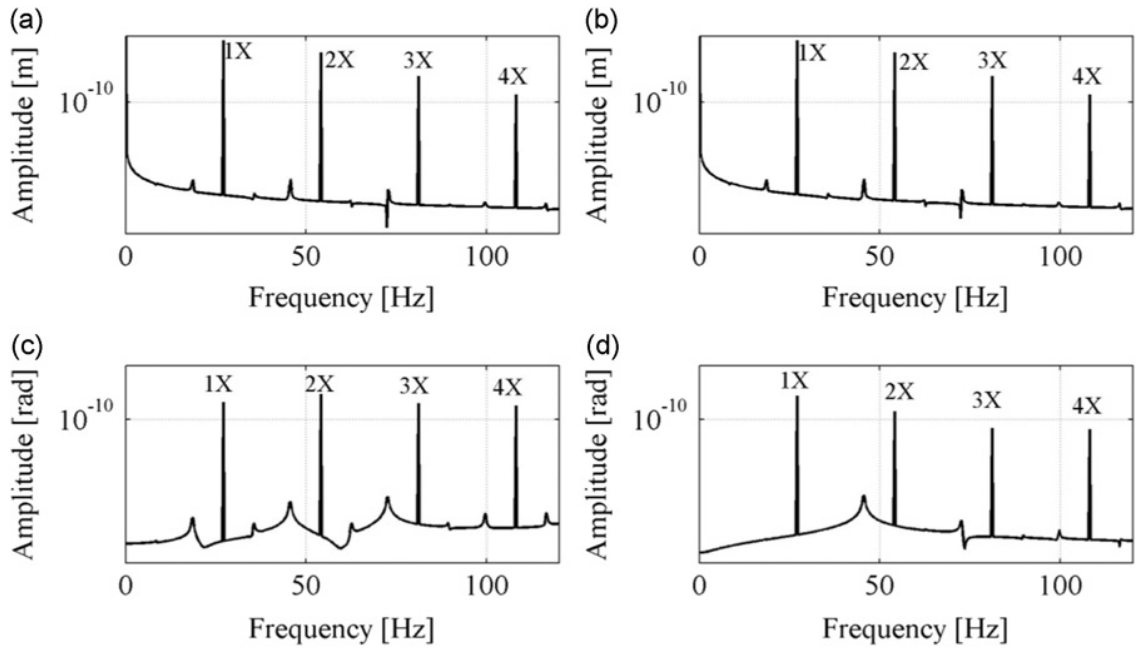

Fig. 15. Cracked rotor frequency spectra of: (a) axial vibration, full stiffness change, (b) axial vibration, half stiffness change, (c) torsional vibration, full stiffness change, (d) torsional vibration, half stiffness change; 25 percent crack, $\beta=45^{\circ}, \varepsilon=10^{-4} \mathrm{~m}$. 
vibrations are shown for full and half stiffness changes. Only synchronous frequency $(1 \times)$ of $27 \mathrm{~Hz}$ and its multiples $(2 \times, 3 \times$, $4 \times$ ) may be observed both in axial and torsional spectra. Comparatively low amplitudes of the axial and torsional vibrations should be pointed out in both cases of the full and half stiffness change. This may be surprising, as one may expect a greater influence of the friction between the lips of the crack on the rotor response. However, such behavior agrees well with the results obtained by others (e.g. [24]).

Similar results to the ones described above are obtained, if different angles between the unbalance and the crack is assumed. The calculations conducted for $\beta=180^{\circ}$ result in almost the same plots, as those presented in Figs. 11-15.

The situation changes dramatically, if larger eccentricity is assumed. The breathing presented in Fig. 16 has been obtained for the 25 percent cracked rotor with the eccentricity of $\varepsilon=10^{-3} \mathrm{~m}$. The crack is almost always open, what can also be observed in Fig. 17d. The amplitude of vibration increases (Fig. 17a and b). The frequency response (Fig. 17c) contains all harmonic multiples $(2 \times, 3 \times, 4 \times)$ of the rotor speed $(1 \times)$, yet the combination frequencies of $18 \mathrm{~Hz}$ and $72 \mathrm{~Hz}$ achieve noticeable values of $6 \times 10^{-7} \mathrm{~m}$ and $2.7 \times 10^{-7} \mathrm{~m}$. Furthermore, the leakage (Fig. 17c) increases significantly. Stiffness coefficients change in a quite different way: $k_{15}, k_{24}, k_{46}$, and $k_{56}$ do not change their sign, and all plots are not so symmetric during a full revolution (compare Fig. 18 with Fig. 13).
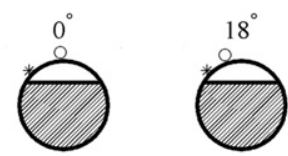

$90^{\circ}$
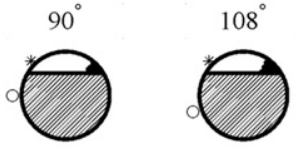

$180^{\circ}$

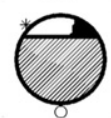

$270^{\circ}$

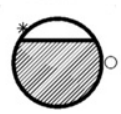

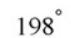

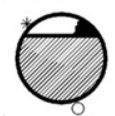

$288^{\circ}$

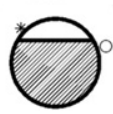

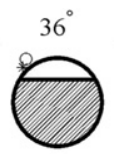

$126^{\circ}$
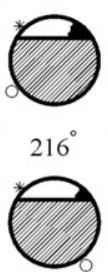

$306^{\circ}$

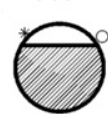

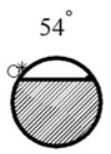

$144^{\circ}$

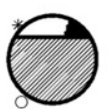

$234^{\circ}$

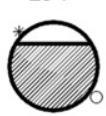

$324^{\circ}$

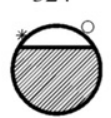

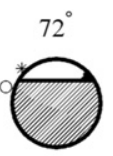

$162^{\circ}$

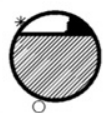

$252^{\circ}$

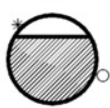

$342^{\circ}$

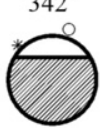

Fig. 16. Crack breathing: 25 percent crack, $\beta=45^{\circ}, \varepsilon=10^{-3} \mathrm{~m}$, full stiffness change.

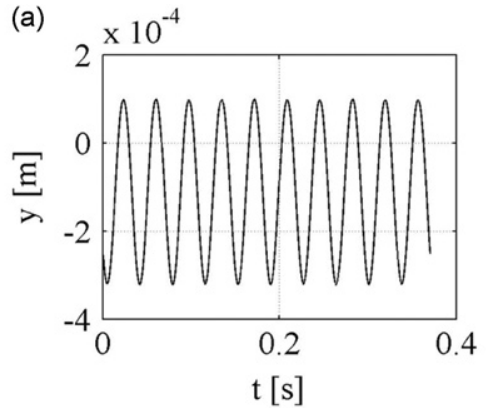

(c)

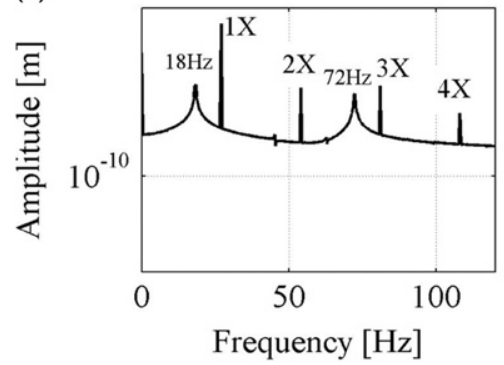

(b)

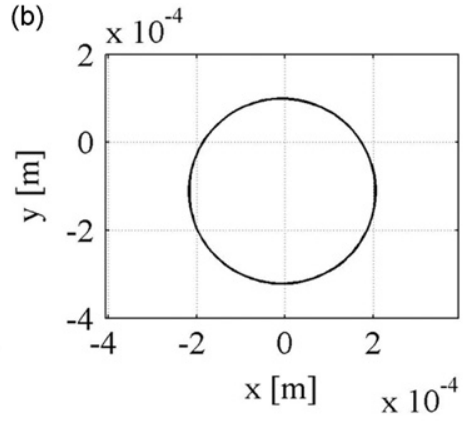

(d)

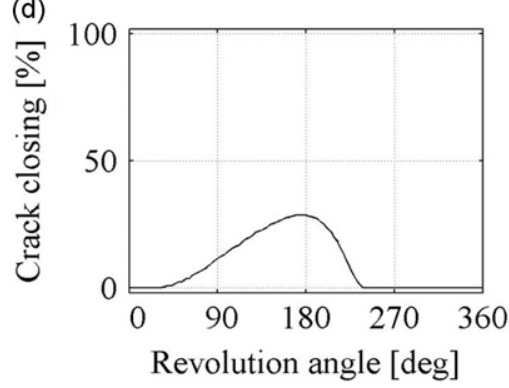

Fig. 17. Cracked rotor response: (a) vertical vibration, (b) orbit, (c) frequency spectrum and (d) crack closing; 25 percent crack, $\beta=45^{\circ}, \varepsilon=10^{-3} \mathrm{~m}$, full stiffness change. 

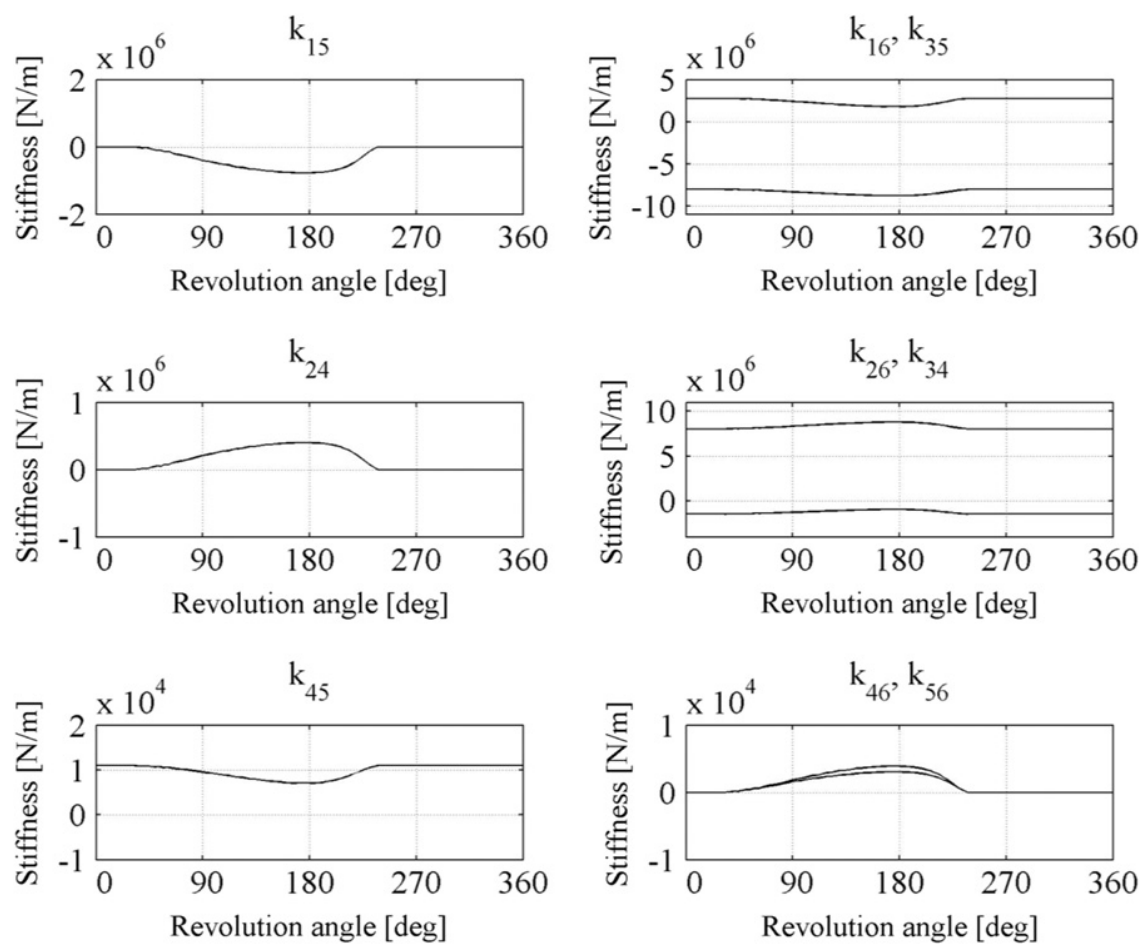

Fig. 18. Cracked area stiffness change: 25 percent crack, $\beta=45^{\circ}, \varepsilon=10^{-3} \mathrm{~m}$, full stiffness change.
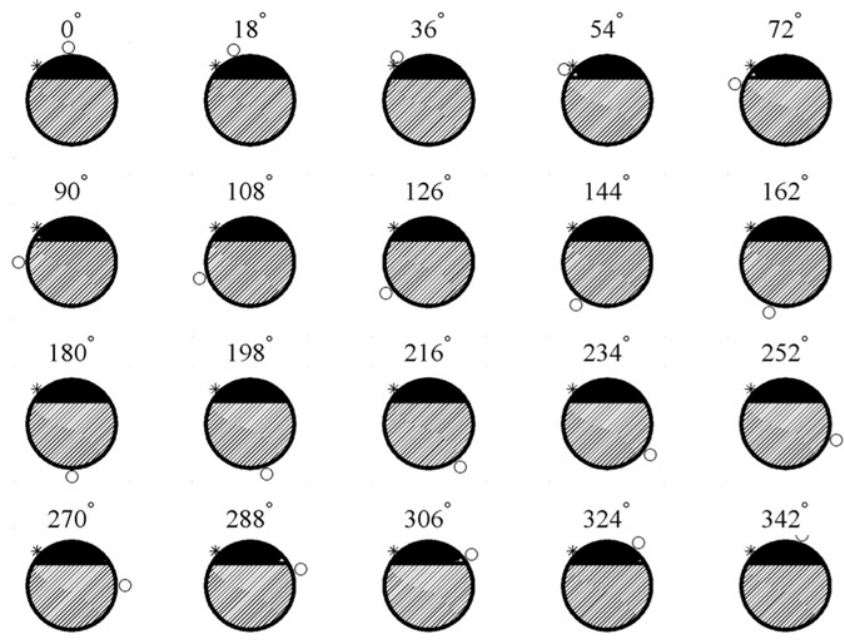

Fig. 19. Crack breathing: 25 percent crack, $\beta=180^{\circ}, \varepsilon=10^{-3} \mathrm{~m}$, full stiffness change.

The situation described above concerns the case, when the angle between the unbalance and the crack is $\beta=45^{\circ}$. Fig. 19 presents the breathing of the same 25 percent cracked rotor with eccentricity $\varepsilon=10^{-3} \mathrm{~m}$, if $\beta=180^{\circ}$. This time the crack remains almost closed during the whole revolution, what results in almost constant values of stiffness coefficients. However, the time response and the orbit (Fig. 20a and b) do not change a lot, when compared with these for $\beta=45^{\circ}$ (Fig. 17a and b). The frequency spectra (Fig. 20c) and Fig. 17c are similar, though the leakage for $\beta=180^{\circ}$ is significantly lower.

For the rotor with the half stiffness change assumed, Figs. 16, 17, 19, 20 look almost the same. Only the plots of stiffness coefficients changes (Fig. 18) are different. This certainly confirms the little influence of the friction between the lips of the crack on its vibration behavior. 

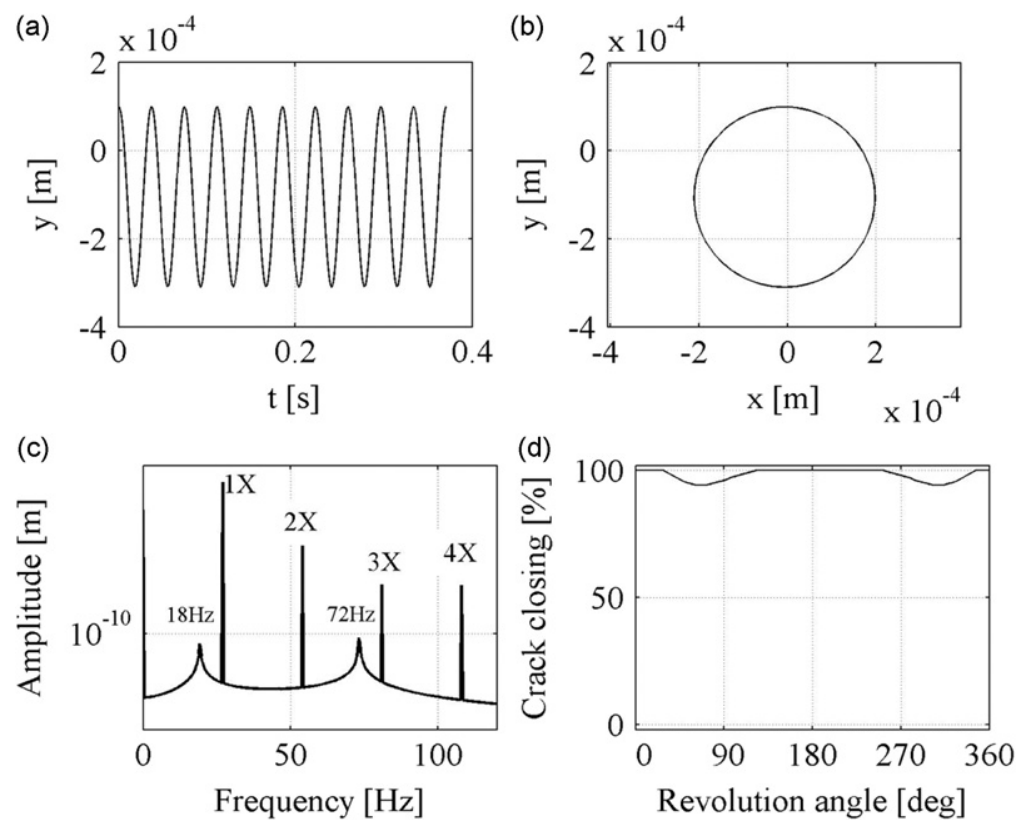

Fig. 20. Cracked rotor response: (a) vertical vibration, (b) orbit, (c) frequency spectrum and (d) crack closing; 25 percent crack, $\beta=180^{\circ}, \varepsilon=10^{-3} \mathrm{~m}$, full stiffness change.
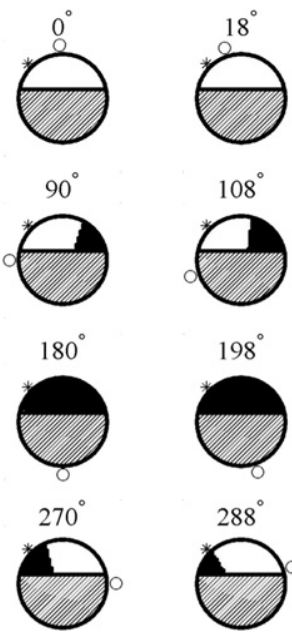
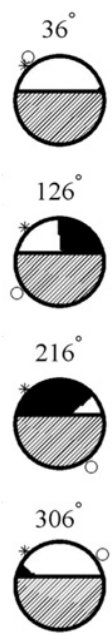
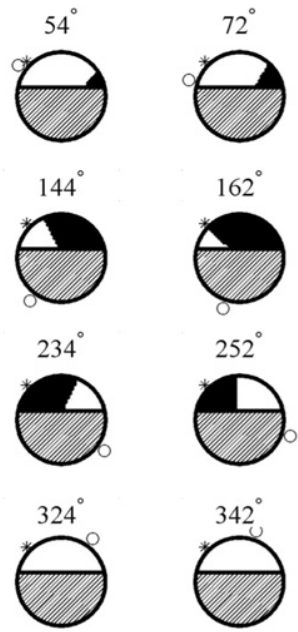

Fig. 21. Crack breathing: 40 percent crack, $\beta=45^{\circ}, \varepsilon=10^{-4} \mathrm{~m}$, full stiffness change.

\subsection{Results for the 40 percent cracked rotor}

For the rotor with the 40 percent deep crack, the results obtained are similar to the ones for the 25 percent deep crack. Figs. 21-23 present selected plots for the 40 percent cracked rotor of eccentricity $\varepsilon=10^{-4}$, and the angle between the unbalance and the crack $\beta=45^{\circ}$. It can be seen that Figs. 21-23 are very close to Figs. 11-13.

\section{Conclusions}

The proposed RFE model of the crack has many advantages:

(a) It can be easily created with the use of a selected number of small spring-damping elements located between the lips of the crack; introducing several subsequent SDEs into the model is simple and can be performed systematically in a way that is natural for the RFE method.

(b) The breathing mechanism can be intuitively explained with the stiffness changes of individual small SDEs; these changes are determined by evaluating deformations of the SDEs. 
(a)

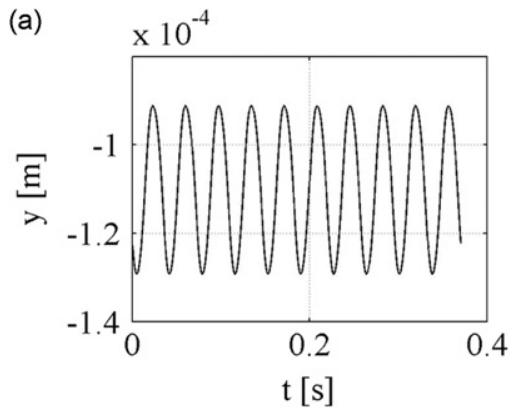

(c)

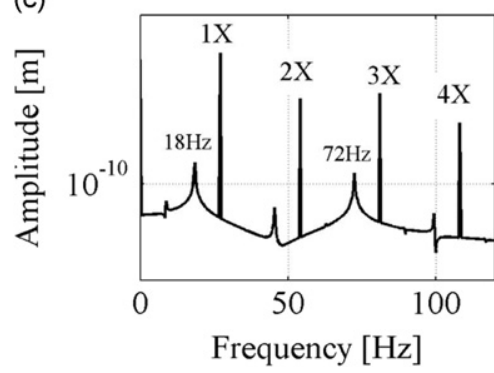

(b)

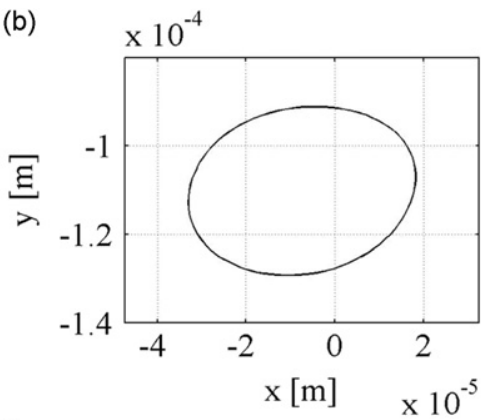

(d)

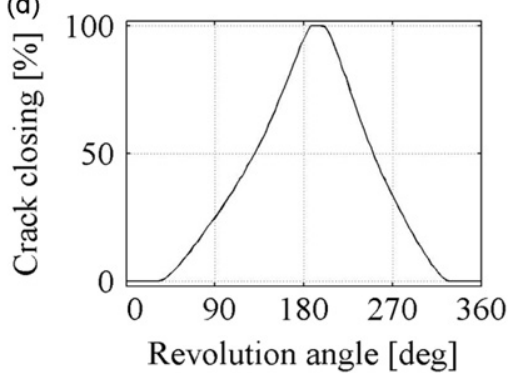

Fig. 22. Cracked rotor response: (a) vertical vibration, (b) orbit, (c) frequency spectrum and (d) crack closing; 40 percent crack, $\beta=45^{\circ}, \varepsilon=10^{-4} \mathrm{~m}$, full stiffness change.
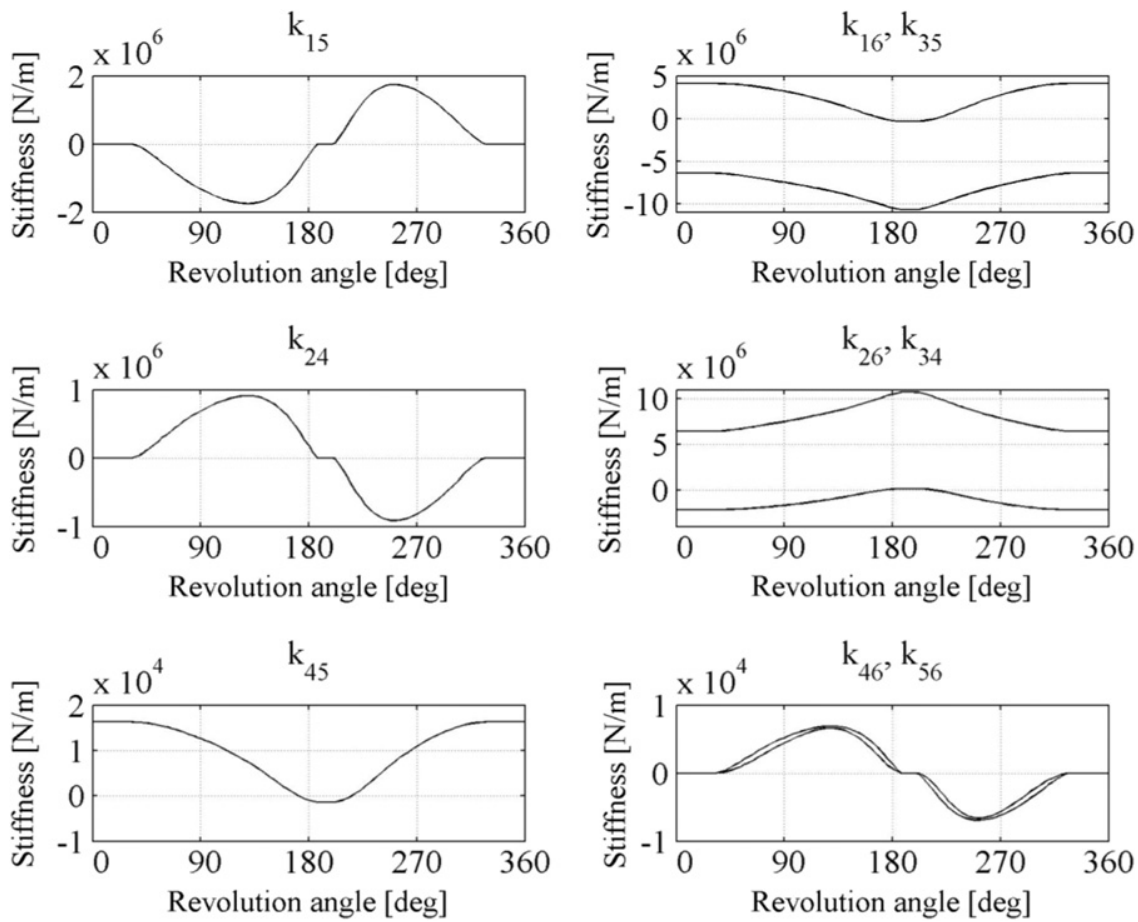

Fig. 23. Cracked area stiffness change: 40 percent crack, $\beta=45^{\circ}, \varepsilon=10^{-4} \mathrm{~m}$, full stiffness change.

(c) Calculations are simple; SDE deformations are calculated using simple matrix operations; no complicated calculations of integrals for flexibilities, of complex formulas for stress intensity factors, etc., are required.

(d) Stiffness matrices of individual SDEs between the crack lips can be modified in such a way that only stiffness changes in selected directions (e.g. stiffnesses for compression, shearing, bending or torsion) can be accounted for; this allows one to easily investigate shearing, sliding or torsional effects between the crack lips.

(e) The calculated transfer function of the uncracked rotor agrees accurately with the experimental data.

(f) Calculation results obtained with the proposed model and with the commercial software agree very well. 
(g) The calculated breathing behavior is similar to that obtained by other researchers [21], who utilized much more complicated and time consuming methods.

Results of calculations show that the breathing behavior of the cracked rotor depends strongly on the value of eccentricity and its angular location. For large eccentricities, the crack may be constantly open (or closed) for some angular locations. For small eccentricities, regardless of their locations, the crack opens and closes (breathes) during the rotation. However, the form of crack breathing has little influence on the vibration response. For all tested situations, the frequency spectra of the vertical vibrations appear almost identical: only the synchronous frequency and its multiples are present. Combination frequencies may appear, yet their amplitudes are very low. Similarly, the amplitudes of the induced, coupled axial and torsional vibrations are low, too.

Surprisingly, the friction between the lips of the crack does not seem to have significant influence on the vibration response. Reducing the shearing and torsional stiffness at the location of the crack alters neither the breathing mechanism, nor the lateral response.

\section{Appendix A. Mass, damping and stiffness matrices}

Using the RFE method the $6 n_{r} \times 6 n_{r}$ mass matrix of a given mechanical structure takes the following block-diagonal form [25]:

$$
\mathbf{M}=\left[\begin{array}{llllll}
\boldsymbol{M}_{1} & 0 & \ldots & \ldots & \ldots & 0 \\
& \mathbf{M}_{2} & 0 & \ldots & \ldots & 0 \\
\ldots & \ldots & \ldots & \ldots & \ldots & \ldots \\
\ldots & \ldots & \mathbf{M}_{r} & \ldots & 0 \\
\ldots & \ldots & \ldots & \ldots & \ldots & \ldots \\
& \ldots & \ldots & \ldots & 0 & \mathbf{M}_{n_{r}}
\end{array}\right]
$$

with blocks of inertia coefficients $\mathbf{M}_{r}$ given by Eq. (1). Note that since blocks $\mathbf{M}_{r}$ are diagonal, matrix $\mathbf{M}$ is also diagonal.

The form of the stiffness matrix is as follows [25]:

$$
\mathbf{K}=\sum_{k=1}^{n_{s}} \tilde{\boldsymbol{K}}_{k}
$$

where the $6 n_{r} \times 6 n_{r} \tilde{\mathbf{K}}_{k}$ matrices are composed of $6 \times 6 n_{r}$ row and $6 n_{r} \times 6$ column stripes and given by

$$
\tilde{\mathbf{K}}_{k}=\left[\begin{array}{lllll}
0 \ldots 0 & 0 & 0 \ldots 0 & 0 & 0 \ldots 0 \\
\ldots & \ldots & \ldots & \ldots & \ldots \\
0 \ldots 0 & 0 & 0 \ldots 0 & 0 & 0 \ldots 0 \\
0 \ldots 0 & \mathbf{K}_{r, r, k} & 0 \ldots 0 & \mathbf{K}_{r, p, k} & 0 \ldots 0 \\
0 \ldots 0 & 0 & 0 \ldots 0 & 0 & 0 \ldots 0 \\
\ldots & \ldots & \ldots & \ldots & \ldots \\
0 \ldots 0 & 0 & 0 \ldots 0 & 0 & 0 \ldots 0 \\
0 \ldots 0 & \mathbf{K}_{p, r, k} & 0 \ldots 0 & \mathbf{K}_{p, p, k} & 0 \ldots 0 \\
0 \ldots 0 & 0 & 0 \ldots 0 & 0 & 0 \ldots 0 \\
\ldots & \ldots & \ldots & \ldots & \ldots \\
0 \ldots 0 & 0 & 0 \ldots 0 & 0 & 0 \ldots 0
\end{array}\right] \text { th row stripe }
$$

and

$$
\begin{array}{r}
\mathbf{K}_{r, r, k}=\mathbf{T}_{r, k}^{T} \mathbf{K}_{k} \mathbf{T}_{r, k}, \quad \mathbf{K}_{p, p, k}=\mathbf{T}_{p, k}^{T} \mathbf{K}_{k} \mathbf{T}_{p, k}, \\
\mathbf{K}_{r, p, k}=-\mathbf{T}_{r, k}^{T} \mathbf{K}_{k} \mathbf{T}_{p, k}, \quad \mathbf{K}_{p, r, k}=\mathbf{K}_{r, p, k}^{T} .
\end{array}
$$

with blocks of stiffness coefficients $\mathbf{K}_{k}$ given by Eq. (2).

The $6 \times 6$ transformation matrices $\mathbf{T}_{r, k}, \mathbf{T}_{p, k}$ take the following forms [25]:

$$
\mathbf{T}_{r, k}=\boldsymbol{\Theta}_{r, k} \mathbf{S}_{r, k}, \quad \mathbf{T}_{p, k}=\boldsymbol{\Theta}_{p, k} \mathbf{S}_{p, k} .
$$

with $6 \times 6$ blocks of rotation coefficients $\boldsymbol{\Theta}_{r, k}, \boldsymbol{\Theta}_{p, k}$ and $6 \times 6$ blocks of coordinates of pinning points $\mathbf{S}_{r, k}, \mathbf{S}_{p, k}$. Block $\boldsymbol{\Theta}_{r, k}$ contains the cosines between the $x_{r, 1}, x_{r, 2}, x_{r, 3}$ and $y_{k, 1}, y_{k, 2}, y_{k, 3}$ frames. Similarly, block $\boldsymbol{\Theta}_{p, k}$ contains the cosines between the $x_{p, 1}, x_{p, 2}, x_{p, 3}$ and $y_{k, 1}, y_{k, 2}, y_{k, 3}$ frames [25]. Block $\mathbf{S}_{r, k}$, contains the coordinates of the pinning point of the $k$ th SDE in the $x_{r, 1}, x_{r, 2}, x_{r, 3}$ frame and block $\mathbf{S}_{p, k}$ the coordinates of the pinning point of the same SDE in the $x_{p, 1}, x_{p, 2}, x_{p, 3}$ frame [25]. 
During the breathing of the crack the blocks of stiffness coefficients $\mathbf{K}_{k}$ change in a way described in Section 4.1 . This results in changes in matrices $\tilde{\mathbf{K}}_{k}$, and consequently in changes in matrix $\mathbf{K}$.

Using the RFE method, the damping matrix can be calculated using the well-known formula, Eq. (6), or it can be obtained using the procedure similar to the procedure of stiffness matrix evaluation. If the latter approach is applied, the $6 n_{r} \times 6 n_{r}$ damping matrix $\mathbf{D}_{D}$ takes the form similar to the stiffness matrix yielded by (A.2) with component matrices $\tilde{\mathbf{D}}_{D, k}$ of the form analogous to (A.3) [25].

The form of the $6 n_{r} \times 6 n_{r}$ block-diagonal gyroscopic matrix is as follows:

with gyroscopic matrices $\mathbf{D}_{G, r}$ given by Eq. (18).

$$
\text { Eq. (18). }
$$

\section{Appendix B. Derivation of the transformation matrix from the local to inertial frames}

From Fig. 8 it is obvious that

$$
\begin{aligned}
& b=q_{r, 3} \cos (\Omega t), \quad c=q_{r, 2} \sin (\Omega t), \\
& d=q_{r, 3} \sin (\Omega t), \quad e=q_{r, 2} \cos (\Omega t),
\end{aligned}
$$

where $q_{r, 2}$ and $q_{r, 3}$ are the coordinates of the center $O^{\prime}$ of the $r$ th RFE in the rotating frame $x_{r, 1}, x_{r, 2}, x_{r, 3}$. From Eq. (B.1) the coordinates $x_{r}$ and $y_{r}$ of the $r$ th RFE's center in an inertial frame $x, y, z$ can be easily obtained as

$$
\begin{gathered}
x_{r}=+(b-c)=-q_{r, 2} \sin (\Omega t)+q_{r, 3} \cos (\Omega t), \\
y_{r}=-(d+e)=-q_{r, 2} \cos (\Omega t)-q_{r, 3} \sin (\Omega t) \leftarrow
\end{gathered}
$$

Since, the axial displacement $z_{r}$ in the inertial frame equals the displacement $q_{r, 1}$ in a rotating frame, Eq. (B.2) can be presented in the following matrix form:

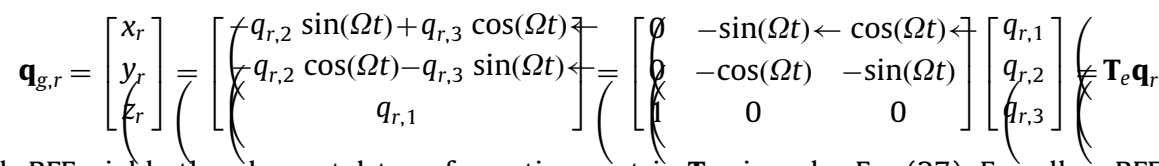

which for the $r$ th RFE yields the elemental transformation matrix $\mathbf{T}_{e}$ given by Eq. (27). For all $n_{r}$ RFEs the form of the transformation matrix $\mathbf{T}_{g}$ utilized in Eq. (26) is straightforward. Note, that $\mathbf{q}_{\mathrm{g}}$ contains only translational displacements of all RFEs.

\section{Appendix C. Stiffness reduction of the cracked shaft spring-damping elements}

As commented in Section 4.1, the dependencies between the stiffnesses of the cracked SDE and the crack depth are nonlinear. These dependencies can be developed as shown below.

Using the RFE approach, stiffnesses of the $k$ th SDE are calculated, as follows [26]:

$$
k_{k, 1}=\frac{E S_{k}}{l_{k}}, \quad k_{k, 2}=\frac{G S_{k}}{\chi l_{k}}, \quad k_{k, 3}=k_{k, 2}, \quad k_{k, 4}=\frac{G J_{k, 1}}{l_{k}}, \quad k_{k, 5}=\frac{G J_{k, 2}}{l_{k}}, \quad k_{k, 6}=\frac{G J_{k, 3}}{l_{k}}
$$

where $S_{k}$ is the cross sectional area; $J_{k, 1}, J_{k, 2}, J_{k, 3}$ are area moments of inertia about axes $y_{k, 1}, y_{k, 2}, y_{k, 3} ; l_{k}$ is the length of the shaft element; and $E, G, \chi$ are Young's modulus, shear modulus and coefficient of cross-section shape (Fig. C1) [26].

Hence, $k_{k, 1}, k_{k, 2}, k_{k, 3}$ depend linearly on cross sectional area and $k_{k, 4}, k_{k, 5}, k_{k, 6}$ depend on area moments of inertia of the crack cross-section. Consequently, Eqs. (22) and (23) should be rewritten as

$$
\begin{gathered}
k_{C, k, j}=\left(1-a_{j}\right) k_{U, k, j} \\
\sum_{k=53}^{53+n_{c}}\left(k_{k, j}=a_{j} k_{U, k, j}\right.
\end{gathered}
$$

where stiffness reductions $a_{1}, a_{2}, a_{3}, a_{4}, a_{5}, a_{6}$ are yielded, as follows:

$$
a_{1}=a_{2}=a_{3}=\frac{S_{C}}{S_{U}}, \quad a_{4}=\frac{J_{C, 1}}{J_{U, 1}}, \quad a_{5}=\frac{J_{C, 2}}{J_{U, 2}}, \quad a_{6}=\frac{J_{C, 3}}{J_{U, 3}}
$$

and $S_{U}, J_{U, 1}, J_{U, 2}, J_{U, 3}$ are cross sectional area and area moments of inertia of the uncracked shaft cross-section; $S_{C}, J_{C, 1}, J_{C, 2}, J_{C, 3}$ are cross sectional area and area moments of inertia of the cracked segment. 


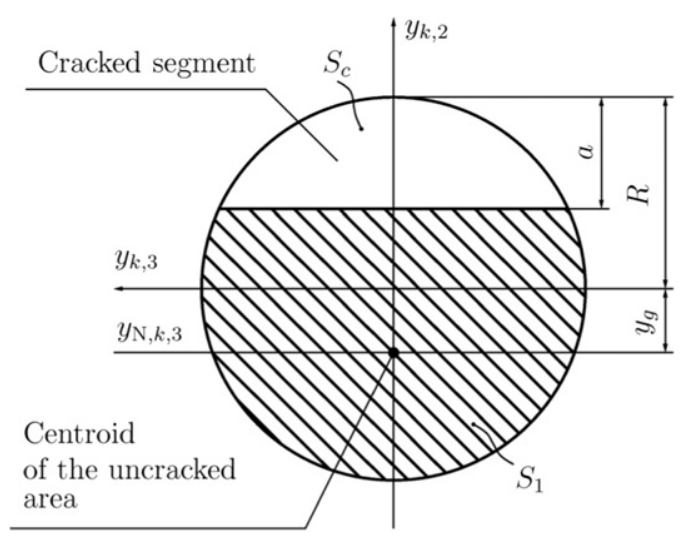

Fig. C1. Schematic diagram of the cracked cross-section.

For the circular cross-section

$$
S_{U}=\pi R^{2}, \quad J_{U, 1}=\frac{\pi R^{4}}{2}, \quad J_{U, 2}=J_{U, 3}=\frac{\pi R^{4}}{4} .
$$

Utilizing the formulas for $S_{C}, J_{C, 2}, J_{C, 3}$ given in [29], i.e.

$$
\begin{gathered}
S_{C}=R^{2}\left(\cos ^{-1}\left(\gamma_{1}\right)-\gamma_{1}\right) \leftarrow \\
J_{C, 2}=\frac{R^{4}}{12}\left(2 \gamma_{1}\left(8 \mu^{2}-8 \mu-3\right) \gamma_{3}+3 \sin ^{-1}\left(2 \gamma_{3}\right)\right) \leftarrow \\
J_{C, 3}=\frac{\pi R^{4}}{8}-\frac{R^{4}}{4}\left(2 \gamma_{1}\left(8 \mu^{2}-8 \mu+1\right) \gamma_{3}+\sin ^{-1}\left(\gamma_{1}\right)\right) \leftarrow
\end{gathered}
$$

the following dependencies can be found:

$$
\begin{gathered}
a_{1}=\frac{\cos ^{-1}\left(\gamma_{1}\right)-2 \gamma_{1} \gamma_{3}}{\pi} \\
a_{5}=\frac{2 \gamma_{1} \gamma_{3}\left(8 \mu^{2}-8 \mu-3\right)+3 \sin ^{-1}\left(2 \gamma_{3}\right)}{3 \pi} \leftarrow \\
a_{6}=\frac{1}{2}-\frac{2 \gamma_{1} \gamma_{3}\left(8 \mu^{2}-8 \mu+1\right)+\sin ^{-1}\left(\gamma_{1}\right)}{\pi} \leftarrow \\
a_{4}=\frac{a_{5}+a_{6}}{2}
\end{gathered}
$$

where

$$
\gamma_{1}=1-2 \mu, \quad \gamma_{2}=\mu(1-\mu), \quad \gamma_{3}=\sqrt{\gamma_{2}},\left(\mu=\frac{a}{2 R}\right.
$$

and $a, R$ are the depth of the crack and the shaft radius, respectively.

For the shaft with a 25 percent deep crack (i.e. for $\mu=25$ percent) the following values can be obtained:

$$
a_{1} \approx 0.20, \quad a_{4} \approx 0.26, \quad a_{5} \approx 0.13, \quad a_{6} \approx 0.40
$$

which means that translational stiffnesses $k_{k, 1}, k_{k, 2}, k_{k, 3}$ should be reduced by 20 percent, while rotational stiffnesses $k_{k, 4}$, $k_{k, 5}, k_{k, 6}$ by 26 percent, 13 percent and 40 percent respectively.

However, stiffness reduction $a_{6}$ given by Eq. (C.11) has been obtained for area moment of inertia $J_{C, 3}$ given by (C.8) about axis $y_{k, 3}$, which is located at the geometrical center of the shaft circular cross section. In fact, during the rotation the shaft is bended about the neutral axis, which is located at the centroid of the uncracked segment, as it is shown in Fig. C1. Thus, to better improve the accuracy, the new stiffness reduction $a_{N, 6}$ should be calculated for the moment of inertia $J_{N, C}$ about neutral axis $y_{N, k, 3}$

$$
a_{N, 6}=\frac{J_{N, C}}{J_{U, 3}}
$$

Utilizing Steiner's theorem, the area moment of inertia $J_{N, C}$ of the cracked segment about axis $y_{N, k, 3}$ can be calculated as

$$
J_{N, C}=J_{C, 3}+S_{C} e^{2}
$$




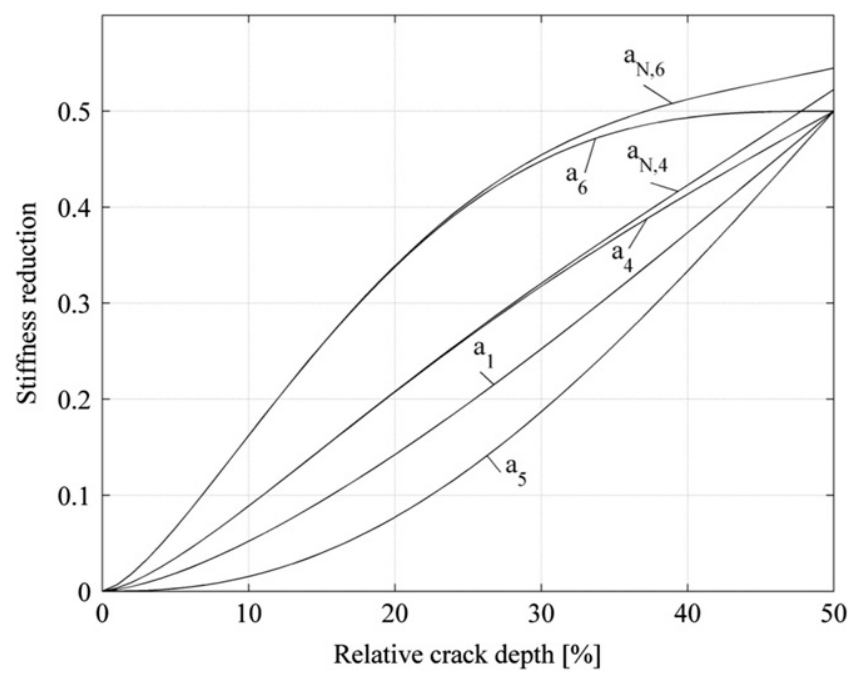

Fig. C2. Stiffness reductions for different crack depths.

The centroid of the cracked segment is shifted by $y_{g}$ along axis $y_{k, 2}$, where [29]

$$
y_{g}=\frac{4 R^{3}}{3 S_{1}} \gamma_{3} \text { and } S_{1}=R^{2}\left(\pi-\cos ^{-1}\left(\gamma_{1}\right)+2 \gamma_{1} \gamma_{3}\right) \leftarrow
$$

Introducing Eqs. (C.16) and (C.15) into Eq. (C.14) the following dependency is yielded:

$$
a_{N, 6}=a_{6}+\frac{128\left(\sin ^{-1}\left(2 \gamma_{3}\right)-2 \gamma_{1} \gamma_{3}\right) \gamma_{2}^{3}}{9 \pi\left(\pi-\cos ^{-1}\left(\gamma_{1}\right)+2 \gamma_{1} \gamma_{3}\right)^{2}}
$$

Consequently

$$
a_{N, 4}=\stackrel{a_{5}+a_{N, 6}}{2}
$$

Respective stiffness reductions for various crack depths are shown in Fig. C2.

\section{References}

[1] M.I. Friswell, J.E.T. Penny, Crack modeling for structural health monitoring, Structural Health Monitoring: An International Journal 1 (2) (2002) $139-148$.

[2] R.A. Gasch, Dynamic behavior of a simple rotor with a cross sectional crack, Vibrations in Rotating Machineries, Proceedings of the International Conference, Institution of Mechanical Engineers, 1976, pp. 123-128.

[3] B. Grabowski, The vibrational behaviour of a rotating shaft containing a transverse crack, in: O. Mahrenholtz (Ed.), Dynamics of Rotors-Stability and System Identification, CISM Courses and Lectures, Vol. 273, Springer, New York, 1984.

[4] I.W. Mayes, W.G.R. Davies, Analysis of the response of a multi-rotor-bearing system containing a transverse crack in a rotor, Journal of Vibration, Acoustics, Stress and Reliability in Design 83 DET 84 (1984) 139-145.

[5] J.T. Sawicki, G.Y. Baaklini, A.L. Gyekenyesi, Coupled lateral and torsional vibrations of a cracked rotor, Proceedings of ASME Turbo Expo 2004 Power for Land, Sea, and Air, Vienna, Austria, June 14-17, 2004.

[6] J.J. Sinou, A.W. Lees, The influence of cracks in rotating shafts, Journal of Sound and Vibration 285 (2005) 1015-1037.

[7] A.D. Dimarogonas, C.A. Papadopoulos, Vibration of cracked shafts in bending, Journal of Sound and Vibration 91 (1983) 583-593.

[8] J.J. Sinou, Experimental response and vibrational characteristics of a slotted rotor, Communications in Nonlinear Science and Numerical Simulation 14 (2009) 3179-3194.

[9] W.M. Ostachowicz, M. Krawczuk, Coupled torsional and bending vibrations of a rotor with an open crack, Archives of Applied Mechanics 62 (1992) 191-201.

[10] K. Darpe, K. Gupta, A. Chawla, Coupled bending, longitudinal and torsional vibrations of a cracked rotor, Journal of Sound and Vibration 269 (2004) 33-60.

[11] J.E.T Penny, M.I. Friswell, Simplified modelling of rotor cracks, Key Engineering Materials 245 (2003) 223-232.

[12] J.T. Sawicki, M.I. Friswell, Z. Kulesza, A. Wroblewski, J.D. Lekki, Detecting cracked rotors using auxiliary harmonic excitation, Journal of Sound and Vibration 330 (2011) 1365-1381.

[13] J.T. Sawicki, D.L. Storozhev, J.D. Lekki, Exploration of NDE properties of AMB supported rotors for structural damage detection, Journal of Engineering for Gas Turbines and Power http://dx.doi.org/10.1115/1.4002908.

[14] R.A. Gasch, A survey of the dynamic behaviour of a simple rotating shaft with a transverse crack, Journal of Sound and Vibration 160 (2) (1993) 313-332.

[15] J.T. Sawicki, X. Wu, G.Y. Baaklini, A.L. Gyekenyesi, , Vibration-based crack diagnosis in rotating shafts during acceleration through resonance, Proceedings of the SPIE 5046 (2003) 1-10.

[16] R.H. Plaut, Parametric, external and combination resonances in coupled flexural and torsional oscillations of an unbalanced rotating shaft, Journal of Sound and Vibration 183 (5) (1995) 889-897.

[17] H.D. Nelson, J.M. McVaugh, The dynamics of rotor bearing systems using finite elements, ASME Journal of Engineering for Industry 98 (1976) 593-600

[18] D. Dimarogonas, S.A. Paipetis, Analytical Methods in Rotor Dynamics, Applied Science Publishers, London, 1983. 
[19] C.A. Papadopoulos, A.D. Dimarogonas, Coupled longitudinal and bending vibrations of a rotating shaft with an open crack, Journal of Sound and Vibration 117 (1987) 81-93.

[20] C. Vare, S. Andrieux, Modeling of a cracked beam section under bending, SMIRT 18, 18th International Conference on Structural Mechanics in Reactor Technology, Bejing, China, 7-12 August, 2005, pp. 281-290.

[21] N. Bachschmid, P. Pennacchi, E. Tanzi, Some remarks on breathing mechanism, on non-linear effects and on slant and helicoidal cracks, Mechanical Systems and Signal Processing 22 (2008) 879-904.

[22] K. Darpe, K. Gupta, A. Chawla, Experimental investigations of the response of a cracked rotor to periodic axial excitation, Journal of Sound and Vibration 260 (2003) 265-286.

[23] A. Vaziri, H. Nayeb-Hashemi, A theoretical investigation on the vibrational characteristics and torsional dynamic response of circumferentially cracked turbo-generator shafts, International Journal of Solids and Structures 43 (2006) 4063-4081.

[24] S. El Arem, Shearing effects on the breathing mechanism of a cracked beam section in bi-axial flexure, European Journal of Mechanics-A/Solids 28 (6) (2009) 1079-1087.

[25] J. Kruszewski, S. Sawiak, E. Wittbrodt, Metoda sztywnych elementów skończonych w dynamice konstrukcji (The Rigid Finite Element Method in Dynamics of Structures), WNT, Warszawa 1999.

[26] E. Wittbrodt, I. Adamiec-Wójcik, S. Wojciech, Dynamics of Flexible Multibody Systems: Rigid Finite Element Method, Springer-Verlag, Berlin, Heidelberg, 2006.

[27] G. Genta, Dynamics of Rotating Systems. Springer-Verlag GmbH 2006.

[28] XLRotor Rotordynamic Software: 〈http://www.xlrotor.com 〉.

[29] M.A. Al-Shudeifat, E.A. Butcher, New breathing functions for the transverse breathing crack of the cracked rotor system: approach for critical and subcritical harmonic analysis, Journal of Sound and Vibration 330 (3) (2011) 526-544.

[30] N.M. Newmark, A method of computation for structural dynamics, ASCE Journal of Engineering Mechanics Division 85 (1959) 67-94. 